\title{
Fibrotic extracellular matrix activates a profibrotic positive feedback loop
}

\author{
Matthew W. Parker, ${ }^{1,2}$ Daniel Rossi, ${ }^{1}$ Mark Peterson, ${ }^{1}$ Karen Smith, ${ }^{1}$ Kristina Sikström, ${ }^{2}$ \\ Eric S. White, ${ }^{3}$ John E. Connett, ${ }^{4}$ Craig A. Henke, ${ }^{1}$ Ola Larsson, ${ }^{2}$ and Peter B. Bitterman ${ }^{1}$
}

\begin{abstract}
${ }^{1}$ Department of Medicine, University of Minnesota, Minneapolis, Minnesota, USA. 2Department of Oncology-Pathology, Karolinska Institutet, Stockholm, Sweden. ${ }^{3}$ Division of Pulmonary and Critical Care Medicine, University of Michigan Medical Center, Ann Arbor, Michigan, USA. ${ }^{4}$ Division of Biostatistics, School of Public Health, University of Minnesota, Minneapolis, Minnesota, USA.
\end{abstract}

\begin{abstract}
Pathological remodeling of the extracellular matrix (ECM) by fibroblasts leads to organ failure. Development of idiopathic pulmonary fibrosis (IPF) is characterized by a progressive fibrotic scarring in the lung that ultimately leads to asphyxiation; however, the cascade of events that promote IPF are not well defined. Here, we examined how the interplay between the ECM and fibroblasts affects both the transcriptome and translatome by culturing primary fibroblasts generated from IPF patient lung tissue or nonfibrotic lung tissue on decellularized lung ECM from either IPF or control patients. Surprisingly, the origin of the ECM had a greater impact on gene expression than did cell origin, and differences in translational control were more prominent than alterations in transcriptional regulation. Strikingly, genes that were translationally activated by IPF-derived ECM were enriched for those encoding ECM proteins detected in IPF tissue. We determined that genes encoding IPF-associated ECM proteins are targets for miR-29, which was downregulated in fibroblasts grown on IPF-derived ECM, and baseline expression of ECM targets could be restored by overexpression of miR-29. Our data support a model in which fibroblasts are activated to pathologically remodel the ECM in IPF via a positive feedback loop between fibroblasts and aberrant ECM. Interrupting this loop may be a strategy for IPF treatment.
\end{abstract}

\section{Introduction}

Organ function depends upon a connective tissue stromal network that imparts topographic integrity by precisely ordering cellular and tissue compartments. The stroma consists of cells and their extracellular matrix (ECM) products. Fibroblasts maintain stromal homeostasis by integrating signals from the ECM that control their function and fate (1-4). Stromal pathology, characterized by pathological fibroblasts and a stiff ECM, is the signature of human diseases ranging from cardiovascular and pulmonary diseases to cancer (5-8), and yet we have only a limited understanding of the mechanisms governing the transition from healthy to diseased stroma $(9,10)$. One central unresolved issue in fibrotic disorders is the extent to which disease progression results from intrinsically diseased fibroblasts, a diseased ECM that corrupts otherwise normal fibroblasts, or a collaboration between the two.

Idiopathic pulmonary fibrosis (IPF) is a model fibrotic disease in which progressive scarring of the lungs leads to death by asphyxiation (11). It is distinguished by the aberrant proliferation of activated fibroblasts and pathological remodeling of the ECM (12). The disease process begins at the base and periphery of the lung in a subpleural distribution (13) and progresses in an upward and inward manner by contiguous spreading to form an uninterrupted fibrotic reticulum that decreases lung compliance and impairs gas exchange $(14,15)$. In IPF, both the fibroblasts and the ECM are profoundly altered. Lung fibroblasts obtained from IPF patients manifest aberrant activation of growth factor signaling pathways, relaxation of cell cycle controls, and alterations in apoptosis regulation (16-19). The ECM in IPF is an inelastic environment containing excessive amounts of connective tissue, matrix metal-

Conflict of interest: The authors have declared that no conflict of interest exists. Citation for this article: J Clin Invest. 2014;124(4):1622-1635. doi:10.1172/JCI71386. loproteinases, growth factors, and morphogens (20-23). Although it is clear that the ECM plays a crucial role in directing cellular processes and maintaining tissue integrity (24-26), the extent to which a pathological ECM can drive a pathological cellular phenotype in IPF is not well defined.

The gene expression pathway can be regulated at multiple levels by transcriptional, posttranscriptional, and posttranslational mechanisms. Translational control is emerging as a principal posttranscriptional mechanism that can substantially affect protein levels genome wide (27). Although fibrotic tissues and fibroblasts have been mainly studied at the steady-state RNA level (commonly designated the "transcriptome," although mechanisms regulating other regulatory steps such as RNA stability also affect steady-state RNA levels), there is evidence for pathologically regulated mRNA translation in fibrotic fibroblasts (28). This raises the possibility that interactions between the ECM and the fibroblast could also affect gene expression at the level of translation.

To explore the relative contribution of the fibrotic ECM and the fibroblast to pathological gene expression, we cultivated primary IPF or control lung fibroblasts on decellularized ECM prepared from IPF or control lungs and conducted genome-wide profiling of both total steady-state and polysome-associated RNA (enriched for mRNAs that are being translated into proteins). Here, we show that the predominant driver of pathological gene expression is the diseased ECM and not the diseased fibroblast and that ECMsensitive genes are primarily modulated at the level of translation. Furthermore, genes translationally activated by the IPF ECM are enriched for ECM proteins found in the IPF lung (e.g., collagen, laminin). This suggests a model for disease progression in which a positive feedback loop between the diseased ECM and fibroblasts propagates the fibrosis, a concept that aligns well with the characteristic pattern of contiguous spreading during IPF progres- 
sion. By searching for shared RNA regulatory elements among the translationally activated ECM genes, we identified miR-29 as a potential regulator of the feedback loop. ECM targets of miR-29 were preferentially activated by the IPF ECM, miR-29c was downregulated in IPF and control fibroblasts by the IPF ECM, and ectopic overexpression of miR-29c rescued this translational activation of ECM genes. Thus, our data provide direct insight into the molecular underpinnings of fibrosis progression in IPF by unveiling an ECM-driven positive feedback loop that can redirect fibroblast ECM gene expression by reducing miR-29 expression, a known potent negative regulator of ECM genes.

\section{Results}

Pathological gene expression in IPF fibroblasts is primarily driven by the $E C M$. To explore the relative contribution of cell origin and ECM origin to gene expression patterns in fibrotic fibroblasts, we cultured IPF or control fibroblasts on IPF or control ECM in a $2 \times 2$ experimental design (using primary fibroblasts from 5 patients with IPF and 5 control patients) (Figure 1A). From each sample, we measured levels of both steady-state and polysome-associated RNA (Figure 1B). To assess whether this in vitro model reflects fibroblast gene expression in vivo, we compared the steady-state RNA profiles from control and IPF fibroblasts on their native ECM (IPF fibroblasts on IPF ECM compared with control fibroblasts on control ECM) with a published dataset examining ex vivo uncultured fibroblasts (29). We tested the hypothesis that those genes up- or downregulated in IPF fibroblasts on IPF ECM relative to control fibroblasts on control ECM are similarly regulated in ex vivo IPF fibroblasts by calculating Cohen's $\kappa$ statistic. This analysis indicated only slight agreement between the two datasets, but greater than would be expected by chance $(\kappa=0.053$, 95\% CI: 0.032-0.075). To further address whether the gene expression observed in our model system corresponds to that observed in vivo, we tested the hypothesis that genes upregulated or downregulated in IPF fibroblasts on IPF ECM are similarly regulated in a published dataset comparing RNA extracted from snap-frozen IPF tissue with RNA extracted from control tissue (30). Notably, the tissue studied contains many cell types, which could potentially dilute differences in gene expression that are specific to fibroblasts. Again, we observed marginal agreement between our data and the in vivo data $(\kappa=0.026,95 \%$ CI: 0.000-0.053). However, even the modest agreement between our data and two external datasets (one ex vivo and the other in vivo) - despite technical limitations due to different cell isolation procedures and a diluted fibroblast signature - supports the idea that our model system captures some of the disease-relevant differences in IPF fibroblast gene expression that occur in vivo.

We next focused on our data from polysome-associated RNA, because data on mRNAs that are translated more closely correspond to protein levels compared with data on steady-state mRNAs $(27,31)$. As expected, the analysis indicated substantial differences between IPF and control cells on their native ECM as judged by an enrichment of low $P$ values (Figure 1C). To determine whether the observed differences were due to fibroblast-intrinsic changes or whether they resulted from the fibroblast receiving cues from a fibrotic ECM, we compared the effect of ECM type (independent of cell origin) with the effect of fibroblast origin (independent of ECM type). Strikingly, more genes were modulated by ECM type than by cell origin (compare Figure 1, D and E; $P<2.2 \times 10^{-16}$ using the Kolmogorov-Smirnov [KS] test). After adjustment of the $P$ values using the Benjamini-Hochberg false discovery rate (FDR) procedure (32), 389 genes showed statistically significant (FDR < 0.2) differences in the level of polysome-associated RNA between ECM types (Supplemental Table 1; supplemental material available online with this article; doi:10.1172/JCI71386DS1). At this FDR threshold, we found that only one gene was linked to cell origin. Note that this is a relatively permissive FDR threshold, allowing up to $20 \%$ false-positives. Although this is not a stringent criterion for identifying particular genes, this analysis revealed that the majority of changes we observed genome wide were driven by ECM type. We obtained similar results when the same analysis was applied to data from steady-state RNA: most of the observed differences were due to ECM type and not to cell origin (Figure 1, $\mathrm{F}-\mathrm{H} ; P<2.2 \times 10^{-16}$ using the KS test).

We found it intriguing that so few genes were linked to cell origin. To determine whether such genes are truly differentially expressed, we compared our results to cell origin-linked genes from one of our prior studies (conducted on synthetic type I collagen matrices) (28). We reasoned that if genes were modulated in the same direction in IPF fibroblasts across both studies, the observed differences were likely genuine. The set of genes with a $P$ value less than 0.05 in both studies (72 genes) indicates moderate agreement between the two studies $(\kappa=0.476$, 95\% CI: 0.269-0.682) (Figure 1I) and supports the supposition that there is indeed a set of genes that are differentially expressed depending on cell origin. To further investigate this issue, we analyzed a published microarray dataset that focused on steady-state RNA differences between IPF and control cells (conducted on tissue culture plastic and therefore expected to be different from the other studies conducted on biological matrices) (33). This dataset also showed only weak evidence of differences between the IPF and control cells (Figure 1J). Thus, the consensus among these studies is that although there are bona fide differences in gene expression between IPF and control fibroblasts, these differences are relatively small compared with the changes induced by the IPF ECM that we observed here.

A principal role for translation in the gene expression response to IPF $E C M$. Because we measured both total steady-state RNA and polysome-associated RNA, we had the opportunity to determine the relative contribution of steady-state RNA levels (reflecting transcription and RNA stability) and translation to the observed ECM-induced gene expression changes. To study translation, levels of polysome-associated RNA have to be corrected for the contribution from other mechanisms in the gene expression pathway (34). This is necessary because genes whose steadystate RNA levels increase will, by mass action alone, also show more RNA associated with polysomes without any regulation of translation. Correction is often done by considering the translational efficiency (TE) ratio (polysome-associated RNA/steadystate RNA). However, this procedure erroneously assumes that an increase in steady-state RNA is noiselessly associated 1:1 with an increase in polysome-associated RNA (leading to increased levels of false-positives and false-negatives when TE is used to compare conditions). We therefore used a more robust correction method called ANOTA (analysis of translational a ctivity) $(34,35)$, which was recently found to better reflect differences in proteomes as compared with TE measures (36). In this method, polysome-associated RNA data are corrected using a linear fit to steady-state RNA data, which allows for the assessment of biological effects at the level of translation. We followed this 
A

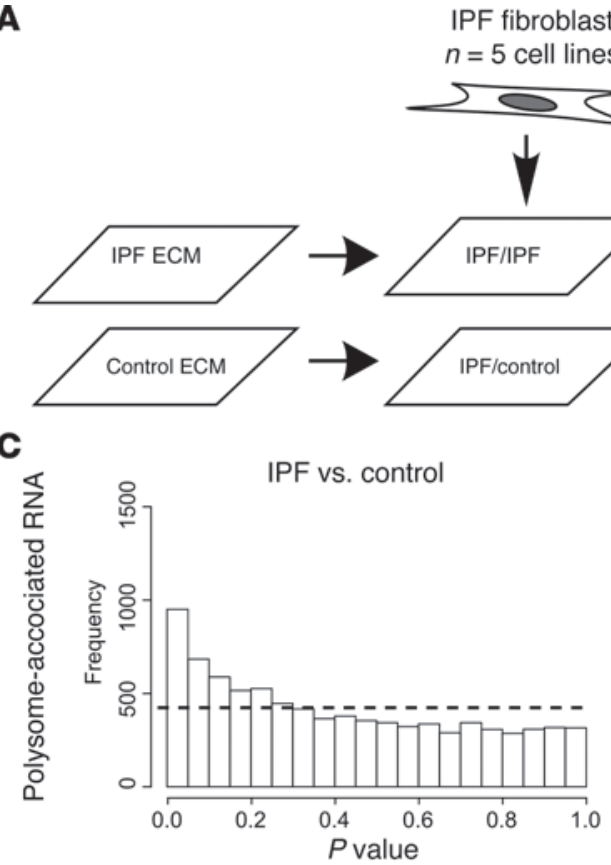

$\mathbf{F}$

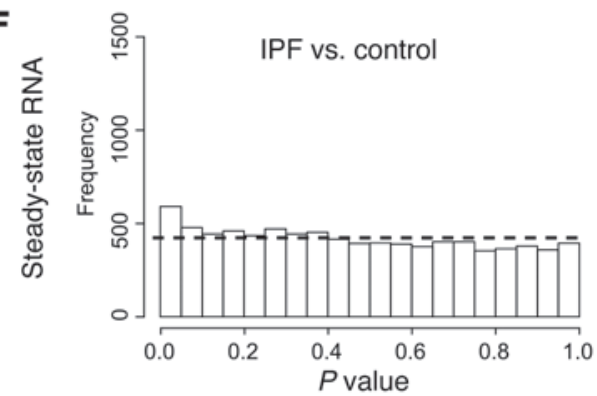

Control fibroblast

$n=5$ cell lines

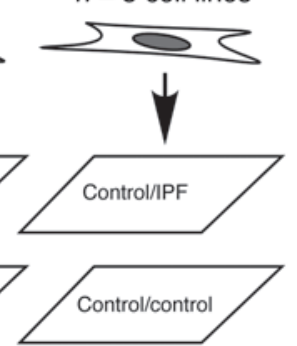

D

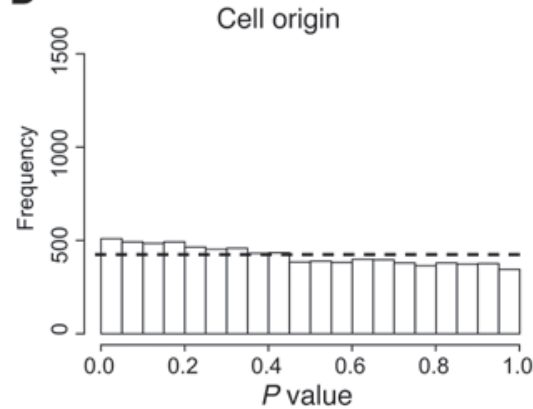

G

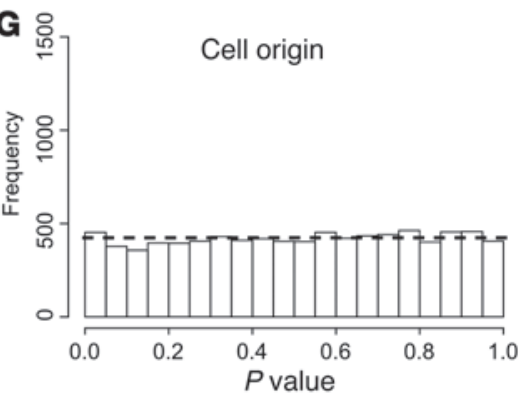

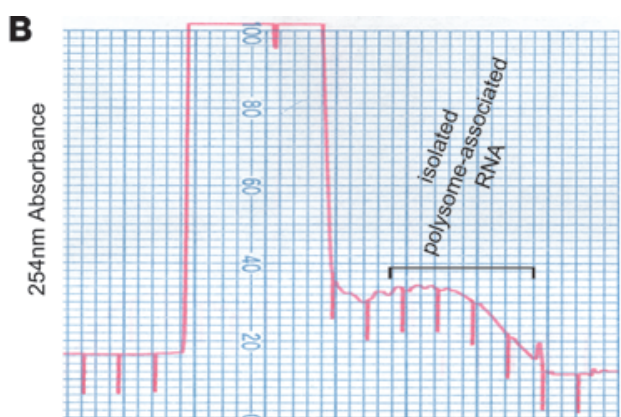

E

Sedimentation
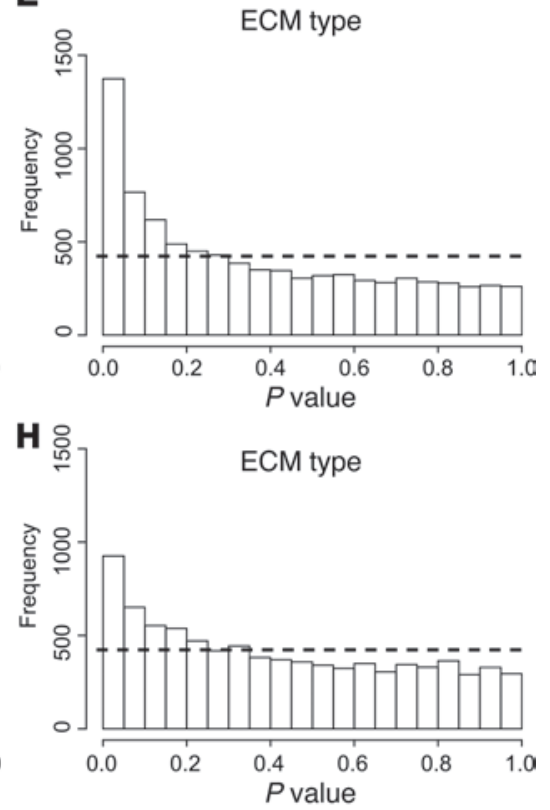
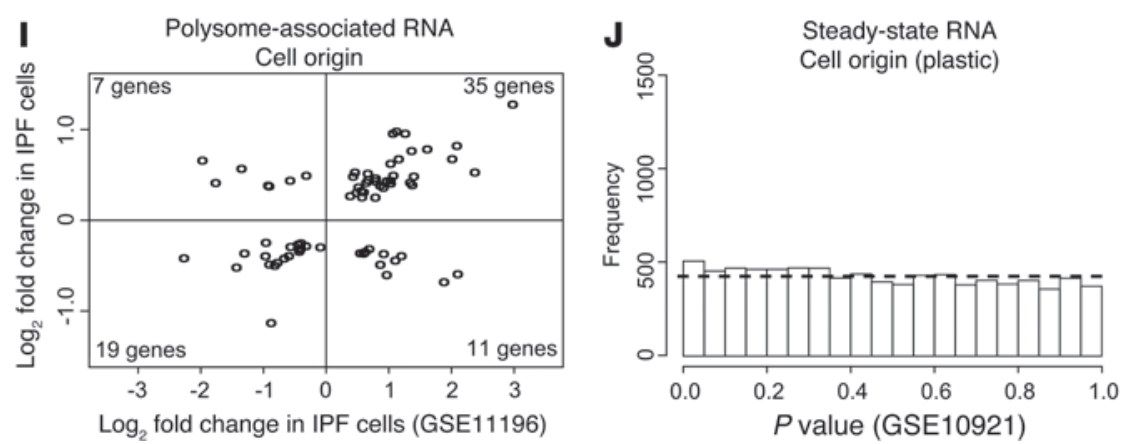

Figure 1

Pathological gene expression in fibrotic fibroblasts is primarily governed by diseased ECM. (A) Experimental design. IPF or control fibroblasts were cultured on IPF or control ECM using a $2 \times 2$ experimental design. (B) Isolation of polysome-associated RNA. Sample polysome tracing shows absorbance at $254 \mathrm{~nm}$ across the sucrose gradient. Transcripts that are translated are enriched among polysome-associated RNA. The portion of the gradient collected to measure polysome-associated RNA levels is indicated. (C-H) Histograms of gene-by-gene $P$ values for different biological comparisons. Dashed lines represent theoretical null distributions. (C-E) Comparison of polysome-associated RNA levels of (C) IPF fibroblasts seeded on IPF ECM and control fibroblasts seeded on control ECM, (D) IPF fibroblasts and control fibroblasts (independent of ECM type), and (E) IPF and control ECM (independent of cell origin). (F-H) Same comparisons as in A-C using steady-state RNA data. (I) Genes that showed cell origin modulation in polysome-associated RNA $(P<0.05)$ in both our current study and in a previous study (microarray data obtained from GSE11196) were collected. Plotted are the fold changes in the previous study ( $x$ axis) and in our current dataset ( $y$ axis). Genes upregulated in one study are more likely to be upregulated in the other $(\kappa=0.476,95 \% \mathrm{Cl}=[0.269,0.682])$. $(\mathrm{J})$ Histogram of $P$ values for comparison of RNA obtained from control and IPF cells grown under standard culture conditions (microarray data obtained from GEO GSE10921). 
method and fit a per-gene linear model to determine the effect of the fibrotic ECM on translation:

$E x_{\text {poly }}=\gamma_{s s} \cdot E x_{s s}+\gamma_{E C M}+\gamma_{c e l l l i n e}+\varepsilon_{\text {within-subject }}$

(Equation 1)

where $E x_{p o l y}$ and $E x_{s s}$ are the data from polysome-associated and steady-state RNA, respectively, $\gamma_{s s}$ is the fitted linear dependence of the polysome-associated RNA on the steady-state RNA, $\gamma_{E C M}$ is the expression difference of cells on the IPF ECM compared with control ECM, $\gamma_{\text {cell line }}$ is the relative expression of the gene in a particular cell line (included to account for the significant variation in gene expression between fibroblasts from different patients), and $\varepsilon_{\text {within-subject }}$ is the within-subject error. The size (and statistical significance) of $\gamma_{E C M}$ reflects the difference in translation induced by IPF ECM. A similar model was used to analyze the cell effects.

In order to determine whether the effects of the fibrotic ECM were primarily due to changes in steady-state RNA levels or to changes in translation, we calculated a cumulative probability distribution of the FDRs for each level (steady-state RNA, polysome-associated RNA and translation [i.e., ANOTA-corrected polysome-associated RNA]) (Figure 2A). As expected, data from polysome-associated RNA (which, in principle, combines the effects from both steady-state RNA levels and translation) showed the greatest number of significantly altered genes. Strikingly, more genes showed low FDRs when we analyzed their translation than did steady-state RNA levels $\left(P=2.6 \times 10^{-8}\right.$ using the KS test). As expected from the low number of genes whose expression depended on cell origin (Figure 1, D and G), the same analysis of the cell origin variable identified very few genes, even at relaxed FDR thresholds (Figure 2B). Thus, we found that the majority of genes that are affected by residence on the diseased ECM were modulated at the level of translation.

As a technical validation step, we next determined whether the observed predominance of translational control among the ECM-induced genes was due to larger fold change differences in data from polysome-associated RNA and not to smaller variances (the latter would indicate that larger measurement noise masked differential expression in steady-state RNA data). A comparison of the fold changes corroborated that the ECM-induced differences in the polysome-associated data are typically higher than in the steady-state RNA data (Figure 2C). The associated variances (residual sum of squares) were also typically higher (Figure 2D). This is expected, because the preparation of polysome-associated RNA involves additional processing, which increases measurement noise. That we found more significant genes despite higher variance is strong evidence that the dominance of translational control is genuine.

Although the majority of changes observed in response to IPF ECM were at the level of translation, there were also genes whose transcript level changed. To assess whether regulation converged on the same genes, we compiled all genes that showed modulation either through translation or at the steady-state RNA level and looked for overlap (FDR $<0.3$, the lowest threshold at which a subset of genes was altered at the steady-state RNA level). A comparison of these genes generated four distinct modules: those genes up- or downregulated either by translation or by steadystate RNA, with only one gene, PRIC285, overlapping (Figure 2E).
Despite the low number of genes modulated by steady-state RNA levels, this suggests that translation and transcription/RNA stability act independently to induce changes to the gene expression profile under these conditions.

Fibrotic ECM activates translation of ECM genes. When we used a gene set enrichment approach (37) to determine whether groups of genes that are associated with a given biological function were translationally upregulated by the IPF ECM, we surprisingly identified several ECM ontologies (e.g., "ECM region," "cell adhesion," and "collagen;" see Table 1). This appeared to be of significance because of the profound changes that the ECM undergoes during IPF disease progression. We therefore further characterized the expression of ECM genes by examining the regulation of all genes in the "ECM region" ontology using data for steady-state RNA or translation. As expected, such genes showed coordinated translational activation by the IPF ECM (Figure 3A, upper panel) and were under stronger regulation than non-ECM genes (Figure 3A, lower panel). Similarly, but to a lesser extent, genes in the ECM region ontology were more translationally regulated by cell origin than non-ECM genes (Figure 3B). The same analysis using data for steady-state RNA indicated that ECM region genes were not regulated more than non-ECM genes by ECM origin (Figure 3C) and that although ECM region genes were more likely to be regulated by cell origin at the steady-state RNA level than non-ECM genes, this regulation was not directional: genes were both upand downregulated (Figure 3D).

This raises the possibility that ECM origin and cell origin together determine the expression pattern for ECM genes by regulating overlapping gene subsets either through changes in translation or in steady-state RNA levels. To assess this, we collected all ECM region genes that were significantly altered $(P<0.05)$ in at least one of the four comparisons (i.e., regulated by cell origin or ECM origin either at the level of steady-state RNA or translation). To separate genes based on which comparisons showed modulation, we used k-means clustering of signed (i.e., upregulation $[+]$ or downregulation [-]) - $\log _{10} P$ values (Figure 4A). As expected from our finding that changes in translational activity and steady-state RNA levels target different subsets of genes (Figure 2E), this clustering revealed a clear segregation of genes into a "translation profile" and a "steady-state RNA profile," with only a small number of genes that did not fit cleanly into either profile. Notably, at the level of translation, most genes that were regulated by cell origin were also regulated by ECM origin, indicating a convergence of cell- and ECM-origin effects for a subset of genes at this control level. In contrast, at the level of steady-state RNA, the majority of genes regulated by cell origin were not regulated by ECM origin. Because the clustering indicated that genes are regulated by either cell origin, ECM origin, or both cell and ECM origin, we compared the fold changes in each of these subgroups. As expected, in the translation profile the cell-regulated genes (Figure 4B, left) and the ECM-regulated genes (Figure 4B, center) had larger cell effects and ECM effects, respectively. In the module of coregulated genes, the ECM and cell effects were similar (Figure 4B, right), indicating that both ECM origin and cell origin shape the expression pattern of these genes to a comparable extent. Of note, gene ontologies associated with ECM remodeling (i.e., "ECM disassembly" and "regulation of angiogenesis") were found to be overrepresented in the ECM-regulated genes, while the two other subgroups had too few genes to allow for efficient analysis of enrichment. The same analysis of fold changes for genes regulated at the steady- 
A

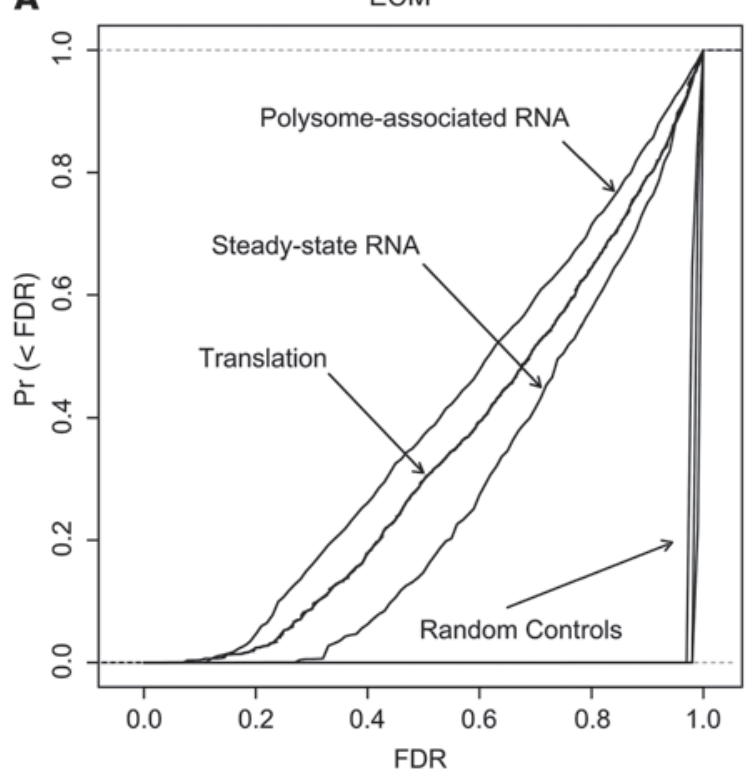

C

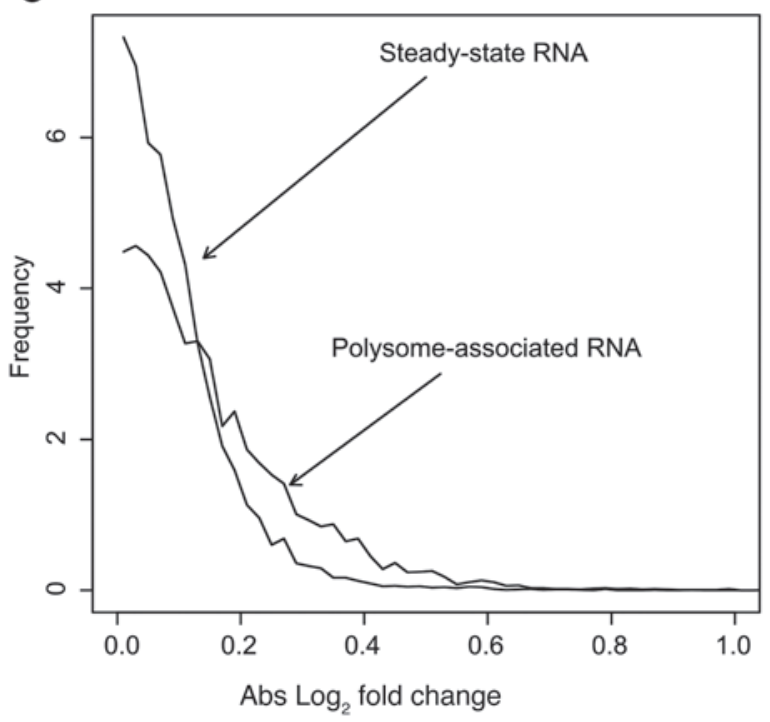

B

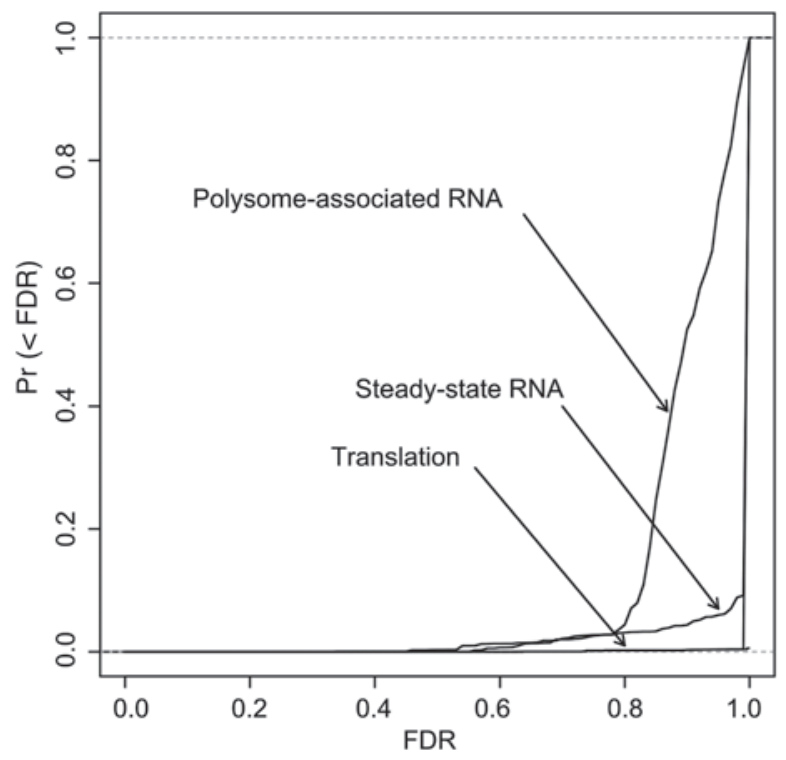

D

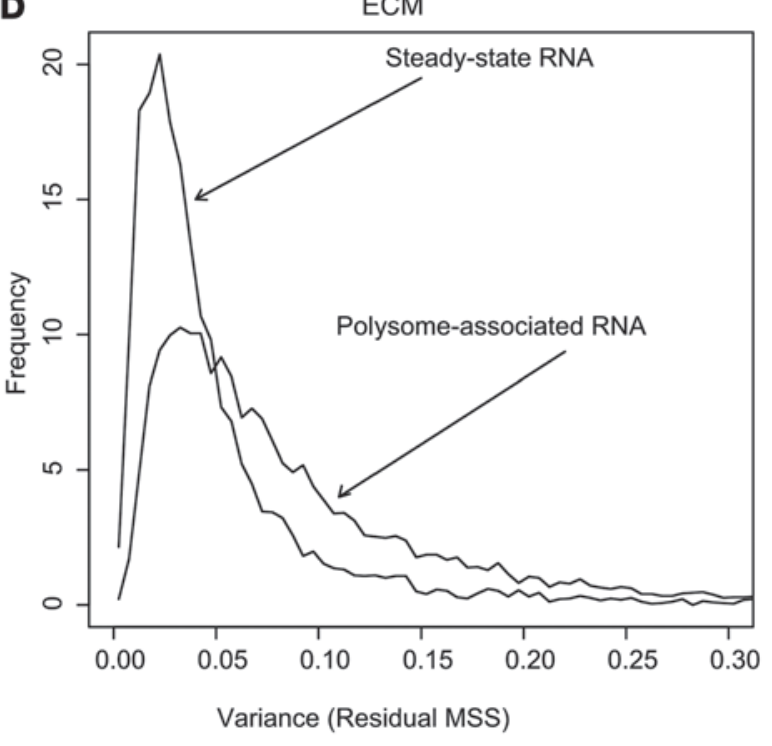

E ECM
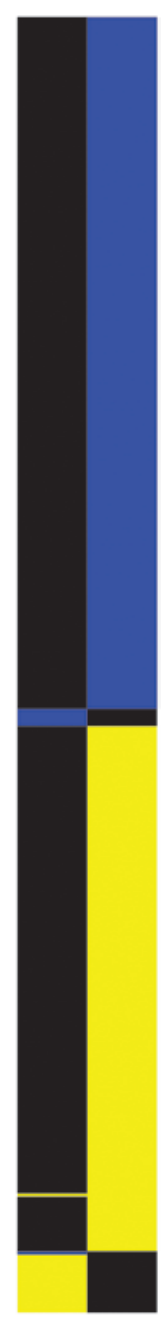

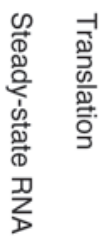

Figure 2

Diseased ECM predominantly affects gene expression by modulating translation. (A and B) Cumulative FDR probability distributions for three control levels: polysome-associated RNA, steady-state RNA, and translation (ANOTA-corrected polysome-associated RNA). Each line indicates the fraction of genes ( $y$ axis) that passed a given FDR threshold ( $x$ axis) for each control level. (A) Comparison between IPF and control ECM. (B) Comparison between IPF and control fibroblasts. (C) Density plot of gene-by-gene fold changes from the ECM origin comparison. (D) Density plot of gene-by-gene variances in comparisons of ECM origin. (E) Heatmap of genes that were upregulated (yellow) or downregulated (blue) at the steady-state RNA or translation level (FDR < 0.3). Pr, probability. Abs, absolute value; MSS, mean sum of squares.

state RNA level mirrored our findings for the translationally regulated group (Figure 4C) and notably identified an "inflammatory response" ontology dependent on ECM origin.

Thus, at each regulatory level (i.e., steady-state RNA and translation), we observed ECM genes that were regulated independently by the ECM and by cell origin. In addition, we observed convergence on a common gene set at the translation level. This implies that although the primary effect in our system is activation of translation induced by the IPF ECM, cell-intrinsic mechanisms also influence the expression of ECM region genes.

ECM genes that are translationally activated by IPF ECM comprise the IPF ECM in vivo. These in vitro results suggest that fibroblasts coordinately induce translation of a set of ECM genes primarily in response to the diseased ECM. It was therefore important to determine whether these ECM genes are found in the IPF ECM in vivo. Our recent proteomic analysis of decellularized IPF ECM 


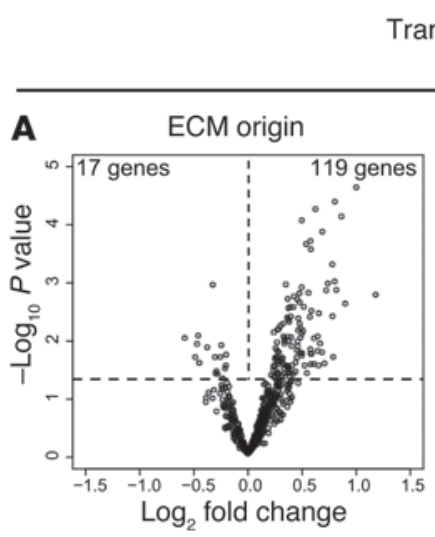

Translation

1

B

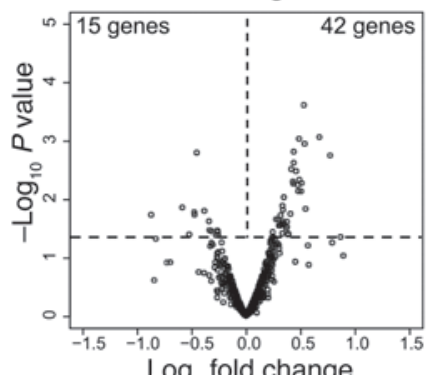

$\log _{2}$ fold change
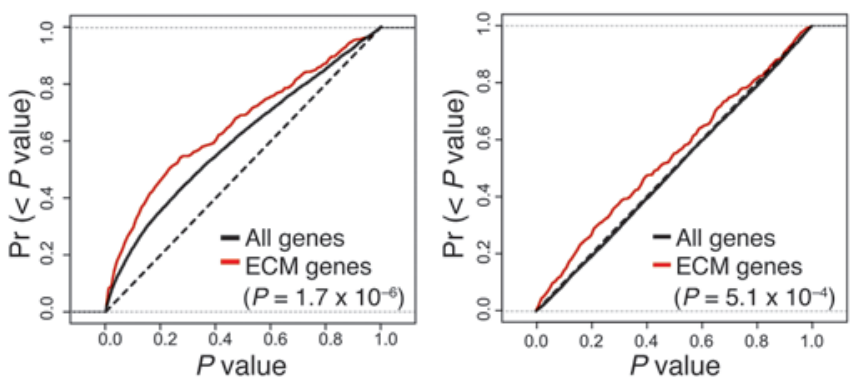

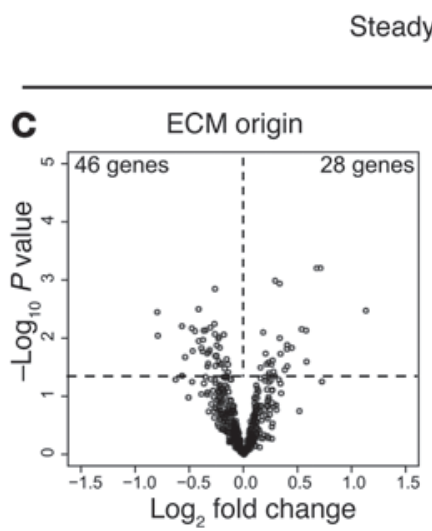

Steady-state RNA

1
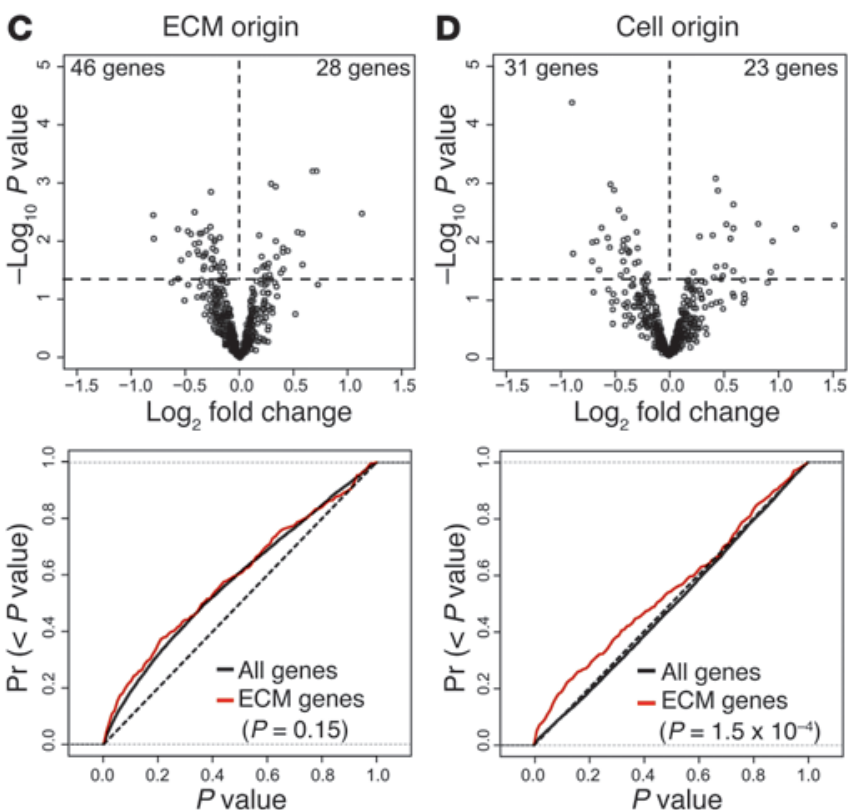

\section{Figure 3}

Diseased ECM coordinately activates the translation of ECM region genes. (A-D) Modulation of genes in the ECM region gene ontology. Upper panels show volcano plots of ECM and cell effects at the translation and steady-state RNA levels. The number of genes up- or downregulated $(P<0.05)$ is indicated. Lower panels show cumulative $P$ value probability distributions from the same comparisons. Dotted line indicates the theoretical null distribution. Solid black line indicates the distribution for all genes. Red line represents the distribution for those genes in the ECM region gene ontology.

(38) identified a panel of proteins that constitute the ECM in IPF. We collected all such genes (termed here IPF-detected proteins) and determined whether they were selectively regulated by the IPF ECM. Indeed, there appeared to be a broad translational upregulation of genes detected in vivo in the IPF ECM in response to decellularized IPF ECM (Figure 5A). To quantify this, we assessed cumulative $P$ value distributions of different nonoverlapping gene subsets (Figure 5, B and C). At the level of translation, we found that ECM genes were more translationally activated by the IPF ECM than all other genes (seen in Figure 3A as well). Strikingly, IPF-detected proteins were in turn significantly more translated than other ECM genes $\left(P=3.9 \times 10^{-5}\right.$, 1 -tailed Mann-Whitney $U$ test). A similar analysis for the cell origin comparison showed that IPF-detected proteins were not more regulated by cell origin than other ECM genes. These findings strongly indicate that the IPF ECM stimulates increased translation of proteins, which are themselves part of the IPF ECM. Moreover, in agreement with identification of ECM origin as the major determinant for disease gene expression, although cell origin has an effect on the synthesis of ECM proteins, these proteins do not appear to be enriched in the IPF ECM in vivo.

A positive feedback loop between the IPF ECM and fibroblasts involves $m i R-29 c$. Our findings are in agreement with the idea that there is a positive feedback loop, wherein the pathological ECM translationally activates ECM genes. There are many mechanisms that can regulate translation (39), including microRNAs, which affect translation and/or RNA stability and thereby protein levels. To search for the possible molecular mechanisms underlying this pos- itive feedback loop, we determined whether the predicted mRNA targets of any microRNA family were overrepresented among the translationally activated ECM genes (those genes with a $P$ value less than 0.05). Indeed, there was one microRNA family (miR-29abcd) whose targets were significantly overrepresented in the group of ECM genes that were translationally activated by the IPF ECM $\left(P=6.28 \times 10^{-5}\right.$, Fisher's exact test). This aligns with recent reports associating miR-29 with IPF $(40,41)$. Indeed, targets of miR-29 were abundant among the IPF-detected proteins that were translationally activated by the IPF ECM (Figure 5A). Our detailed analysis showed that among all IPF-detected proteins, those containing miR-29 targets were significantly more likely to be translationally regulated by the IPF ECM than those lacking targets $(P=0.02$, 1-tailed Mann-Whitney $U$ test; Figure 5B). In contrast, we did not observe any cell origin regulation by miR-29 (Figure 5C). Moreover, we did not find regulation of IPF-detected proteins or miR-29 targets at the steady-state RNA level (Supplemental Figure 1), suggesting that translation is the primary mechanism for regulation downstream of miR-29 under these conditions.

These data indicate that reduced expression of miR-29 in response to IPF ECM could mechanistically explain the positive feedback loop between fibroblasts and the pathological ECM (because microRNAs negatively regulate gene expression). To investigate this, we quantified the levels of the three human forms of the miR-29 family (miR-29a, miR-29b, and miR-29c) in vitro using the $2 \times 2$ experimental model (Figure 1A). Similarly to our analysis in Figure 5, B and C, we compared both the effect of IPF ECM (independent of cell origin) and IPF cell origin (independent 

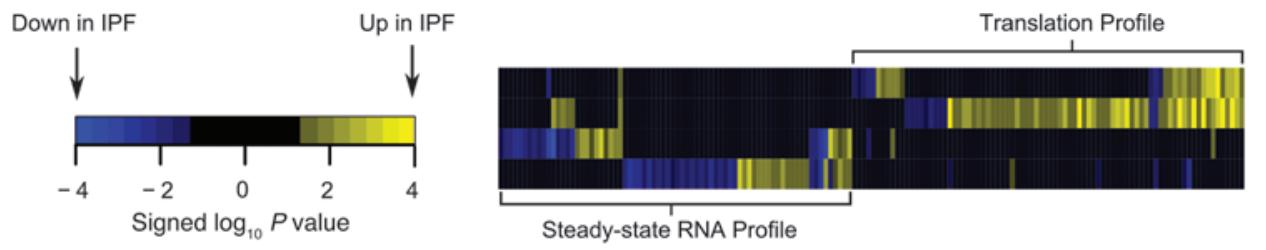

\begin{tabular}{l|l} 
Cell & Translation
\end{tabular}

\begin{tabular}{l|l} 
Cell & Steady-state RNA \\
ECM &
\end{tabular}

Steady-state RNA Profile

B

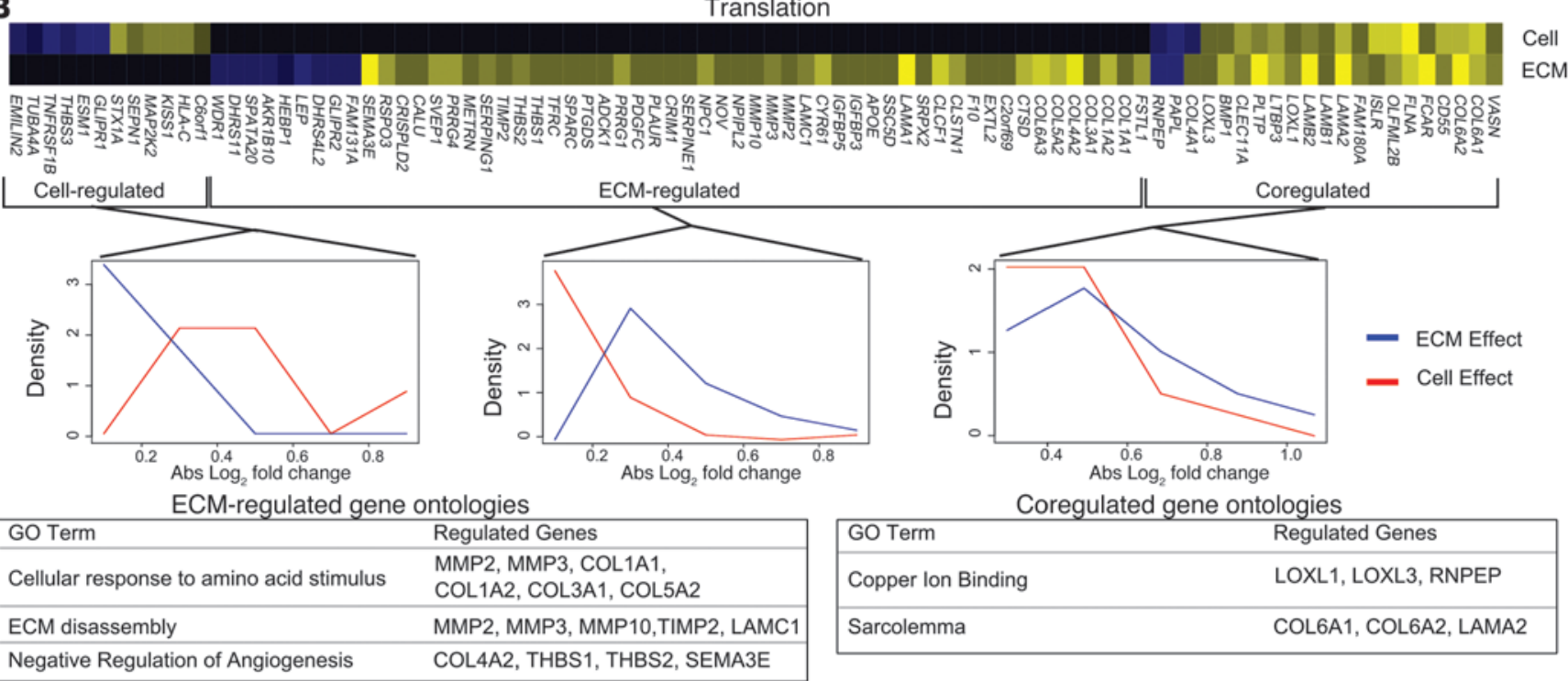

C Steady-state RNA
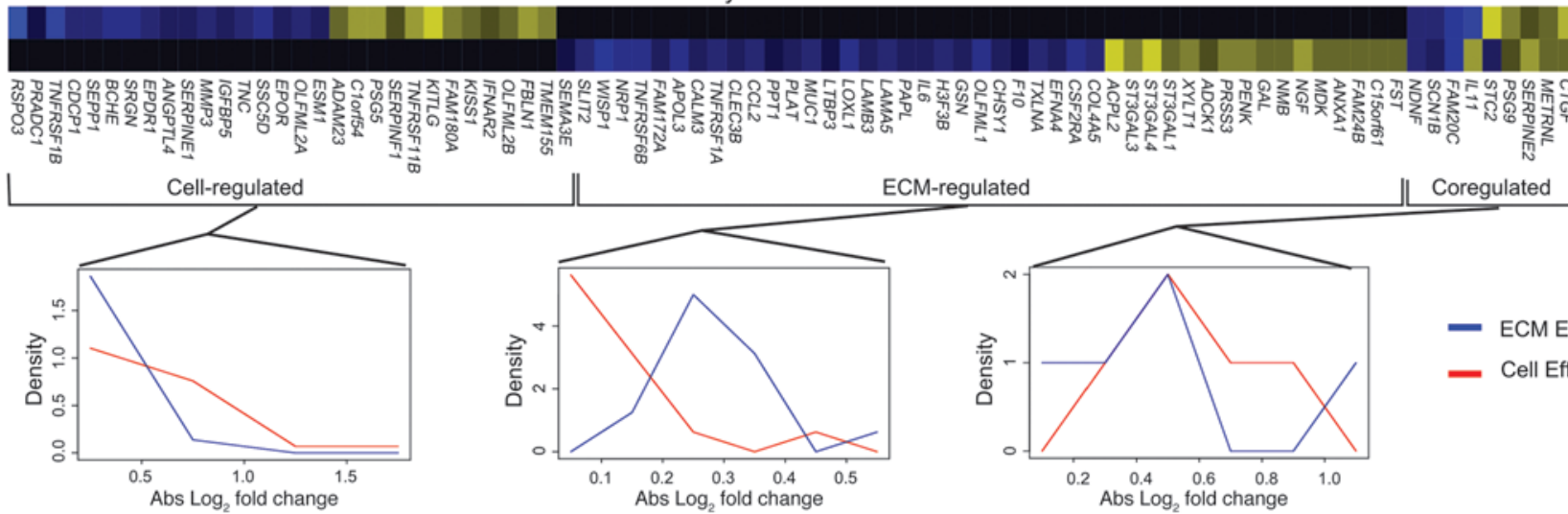

ECM-regulated

Coregulated

ECM-regulated gene ontologies
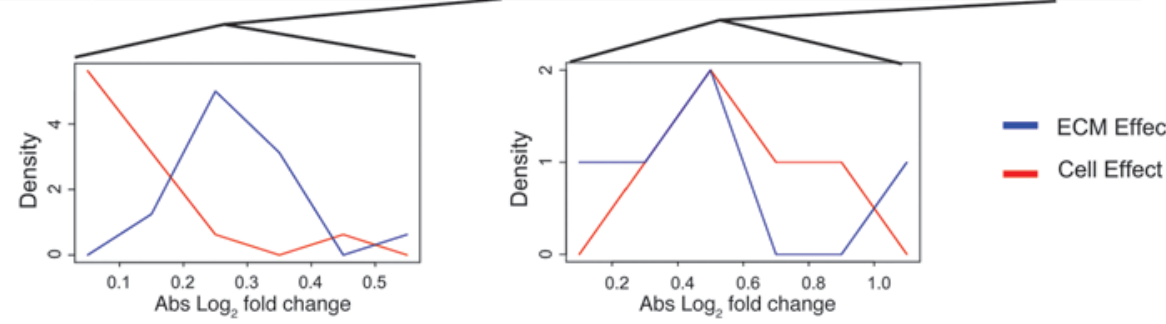

Coregulated gene ontologies

\begin{tabular}{|ll|}
\hline GO Term & Regulated Genes \\
\hline Inflammatory response & ANXA1, IL6, NGF, CCL2, \\
& NGF, TNFRSF1A, APOL3 \\
\hline O-Glycan Processing & MUC1, ST3GAL1, ST3GAL3, ST3GAL4 \\
\hline
\end{tabular}

\begin{tabular}{|ll|}
\hline GO Term & Regulated Genes \\
\hline Glycosaminoglycan binding & SERPINE2, NDNF \\
\hline
\end{tabular}

Figure 4

Converging and independent modulation of ECM gene translation is dependent upon ECM and cell origins. (A) Translation (ANOTA-corrected) and steady-state RNA profiles of genes in the ECM region gene ontology that were differentially expressed $(P<0.05)$ in any comparison. Values are log 10 $P$ values (yellow denotes upregulation in IPF, blue denotes downregulation). (B) Close-up of the translation profile. Genes are divided into three categories: cell-regulated, ECM-regulated, and coregulated. Density plots of the absolute fold changes induced by each biological variable are shown. Selected gene ontologies that are overrepresented $(P<0.01$, calculated using Fisher's exact test) are shown (see Supplemental Table 2 for the complete list). There were no significantly overrepresented gene ontologies in the cell-regulated group. (C) Same analysis for the steady-state RNA profile.

of ECM type). Consistent with the increased translation observed for IPF-detected proteins on the IPF ECM, we found that the level of miR-29c was reduced $(P=0.031)$ and that the other two miR-29 forms trended downward on the IPF ECM (Figure 5D). Moreover, consistent with the lack of any regulation of miR-29 by cell ori- gin, none of the three miR-29 forms were significantly regulated between the two cell origins (Figure 5E). Thus, taken together with our genome-wide data indicating that miR-29 targets are upregulated, modulation of miR-29 expression may partly explain the increased translation of ECM genes on the IPF ECM. To func- 
A
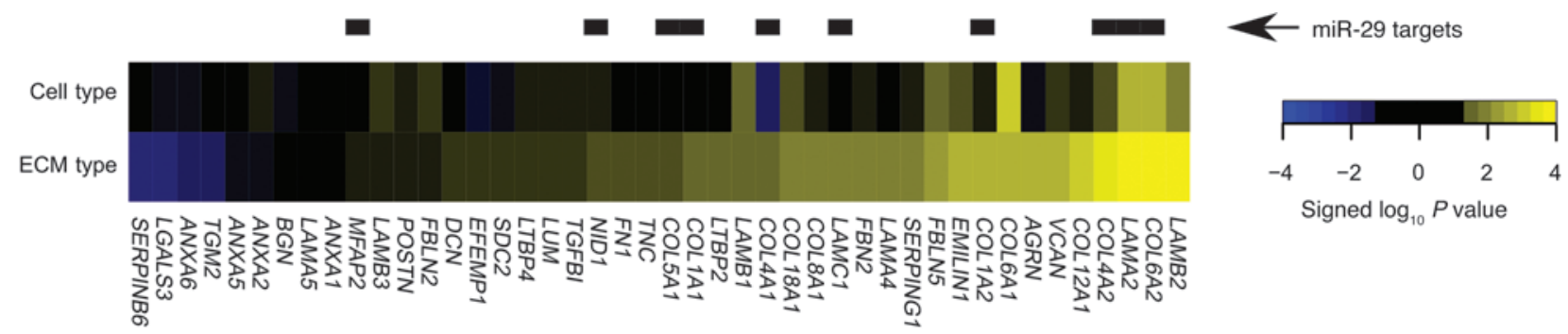

B
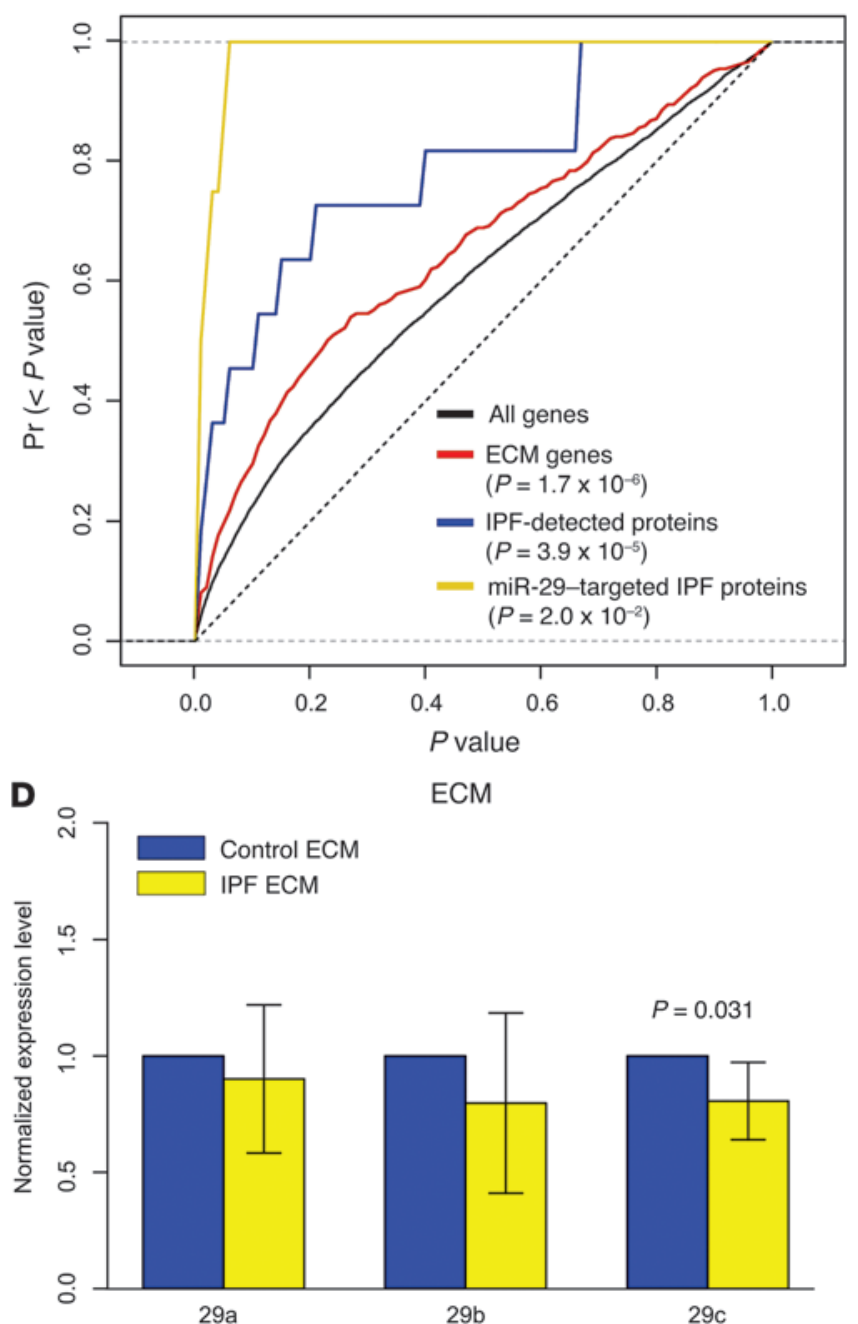

C

Cell

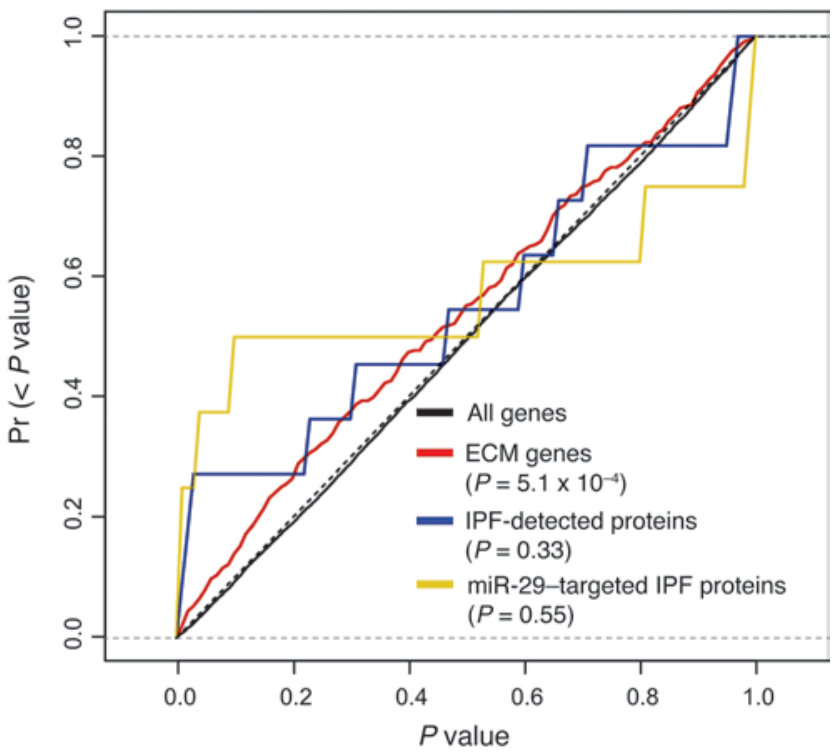

E

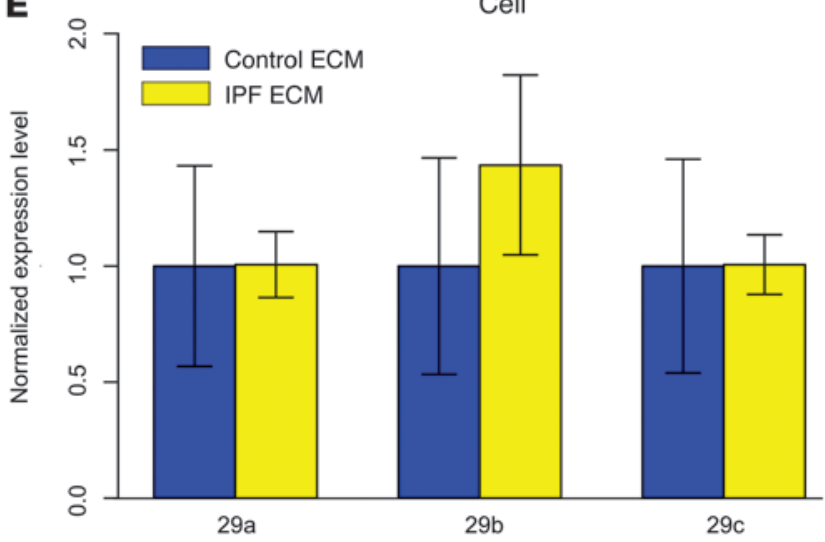

Figure 5

A positive feedback loop between diseased ECM and the fibroblast involves modulation of miR-29 expression. (A) Translation profile of genes whose protein products are present in IPF lung ECM. Shown are $-\log _{10} P$ values from the cell origin and ECM origin comparison (yellow denotes upregulation in IPF, blue denotes downregulation). miR-29 targets are designated by solid bars above the heatmap. (B and C) Cumulative $P$ value probability distributions for (B) ECM origin and $(\mathbf{C})$ cell origin are shown. Dotted line represents the theoretical null distribution. Black line represents all genes except ECM region genes. Red line represents all ECM genes except those detected in the IPF lung (KS $P$ value for comparison with "All genes"). Blue line represents all genes from the IPF lung except miRNA-29 targets (KS $P$ value for comparison with "ECM genes"). Yellow line represents all IPF-detected miR-29 targets (KS $P$ value for comparison with "IPF-detected proteins"). (D and $\mathbf{E}$ ) Levels of miR-29 species were quantified using qPCR. In all comparisons, the arbitrary units were normalized to the control level. The levels of miR-29c between control and IPF ECM were significantly altered $(P=0.031)$. It should be noted that the ECM comparison is paired so the single error bar represents the standard error between the paired differences. 


\begin{tabular}{|c|c|c|c|}
\hline GO term & & FDR & Set size \\
\hline G0:0005576 & Extracellular region & $1.02 \times 10^{-12}$ & 491 \\
\hline G0:0005615 & Extracellular space & $3.47 \times 10^{-9}$ & 238 \\
\hline G0:0031012 & ECM & $2.38 \times 10^{-8}$ & 83 \\
\hline G0:0005789 & Endoplasmic reticulum membrane & $2.13 \times 10^{-4}$ & 371 \\
\hline G0:0005887 & Integral to plasma membrane & $4.21 \times 10^{-4}$ & 288 \\
\hline G0:0005578 & Proteinaceous ECM & $4.37 \times 10^{-4}$ & 58 \\
\hline G0:0004872 & Receptor activity & $6.45 \times 10^{-4}$ & 278 \\
\hline G0:0007155 & Cell adhesion & $1.01 \times 10^{-3}$ & 184 \\
\hline G0:0005178 & Integrin binding & $2.17 \times 10^{-3}$ & 36 \\
\hline G0:0005201 & ECM structural constituent & $2.20 \times 10^{-3}$ & 27 \\
\hline G0:0005788 & Endoplasmic reticulum lumen & $2.30 \times 10^{-3}$ & 60 \\
\hline G0:0005604 & Basement membrane & $3.80 \times 10^{-3}$ & 33 \\
\hline G0:0005509 & Calcium ion binding & $4.46 \times 10^{-3}$ & 211 \\
\hline G0:0007040 & Lysosome organization & $1.34 \times 10^{-2}$ & 20 \\
\hline G0:0005581 & Collagen & $1.58 \times 10^{-2}$ & 28 \\
\hline G0:0007411 & Axon guidance & $1.87 \times 10^{-2}$ & 147 \\
\hline G0:0000139 & Golgi membrane & $1.90 \times 10^{-2}$ & 250 \\
\hline G0:0006865 & Amino acid transport & $1.90 \times 10^{-2}$ & 13 \\
\hline G0:0008201 & Heparin binding & $2.27 \times 10^{-2}$ & 46 \\
\hline G0:0009986 & Cell surface & $2.27 \times 10^{-2}$ & 127 \\
\hline G0:0005102 & Receptor binding & $2.58 \times 10^{-2}$ & 100 \\
\hline G0:0005624 & Membrane fraction & $3.39 \times 10^{-2}$ & 226 \\
\hline G0:0043687 & Posttranslational protein modification & $4.38 \times 10^{-2}$ & 111 \\
\hline G0:0050900 & Leukocyte migration & $4.75 \times 10^{-2}$ & 47 \\
\hline
\end{tabular}

The list of genes ranked by change induced in translation by ECM type (signed - $\log _{10}$ $P$ value) was analyzed using GAGE (37) to identify gene ontologies that are activated through translation. $\mathrm{GO}$, gene ontology.

effects. Regardless, our data indicate a role for miR29 in the fibrotic feedback loop; miR-29c overexpression can abrogate pathological upregulation of miR29-targeted ECM genes induced by IPF ECM.

\section{Discussion}

Despite the profound changes that the ECM undergoes in fibrosis, there has been a paucity of studies exploring how these changes affect fibroblast gene expression genome wide. Here, we addressed this gap by seeding fibroblasts originating from IPF or control lungs into IPF ECM or control ECM and measuring the resultant gene expression. We thereby determined changes in gene expression that were intrinsic to the IPF fibroblast and changes that resulted from the fibroblast responding to the diseased ECM. Surprisingly, ECM origin had a greater impact on gene expression than did cell origin, and the majority of these changes occurred at the translation level and not at the transcription/RNA stability level. This is in agreement with emerging data positioning translation as the principal posttranscriptional mechanism $(27,42)$.

The issue of cell-intrinsic versus microenvironment-dependent gene expression is complex. We endeavored in this study to examine genome wide how the interaction between fibroblasts and the ECM differs when the same fibroblasts are cultured on control ECM compared with when they are cultured on fibrotic ECM. The isolation of fibroblasts from the lung and their subsequent expansion, although necessary to conduct such experiments, could potentially mask differences between IPF

tionally assess this possibility, we tested whether ectopic expression of miR-29 could rescue the translation of ECM genes in IPF fibroblasts cultured on IPF ECM. For this, IPF fibroblasts infected with a lentivirus expressing either an hsa-miR29c construct or a scrambled control were cultured for 18 hours on diseased ECM (Figure 6A), and polysome-associated RNA levels for four miR-29 target genes that were translationally activated by diseased ECM were quantified by quantitative PCR (qPCR). Cells overexpressing hsa-miR29c showed an approximate $50 \%$ increase in miR-29c expression on IPF ECM compared with the levels observed in cells receiving the scrambled construct on IPF ECM (Figure 6B), an increase in miR-29c expression that is similar to that observed for endogenous miR-29c on control ECM relative to IPF ECM (Figure 5D). Whereas cells treated with control virus showed aberrantly activated translation of four genes with target sites for miR29 (after normalization to the GAPDH control) when cultured on IPF ECM, cells with ectopic expression of miR-29 showed translation close to the baseline level observed on control ECM (Figure 6C). In contrast, four control genes lacking a miR-29 target site that were upregulated by the IPF ECM did not return to baseline with miR-29 overexpression (Figure 6D). Interestingly, two ECM genes, COL12A1 and $A G R N$, were upregulated by the IPF ECM and returned toward baseline when miR-29c was overexpressed despite not being annotated as harboring any miR-29 targets (Figure 6E). This indicated incompleteness of the miR-29 annotation and/ or that miR-29 may be responsible for some of the upregulation observed even among nontarget ECM genes through secondary and control fibroblasts that were present in vivo, an effect that may be partially responsible for the relatively few cell-intrinsic differences that we observed. Nonetheless, agreement with previous ex vivo and in vivo datasets indicates that the differences we did observe are likely to be trustworthy. Because of these issues, we focused on the relatively larger differences in gene expression induced by fibrotic ECM.

We found that ECM genes targeted by miR-29 were selectively upregulated in response to the fibrotic ECM, spotlighting miR-29 as a critical lynchpin in the pathological interaction between the ECM and the fibroblast. This finding is consistent with a general role for miR-29 in fibrosis; expression of this microRNA is not only low in IPF (43) but in other fibrotic diseases as well (44-46). In fact, a recent study has shown that miR-29 gene transfer into the lungs of mice with bleomycin-induced lung fibrosis can arrest - but not reverse - the progression of fibrosis (47). In addition, downregulation of miR-29 is known to activate the synthesis of ECM products by lung fibroblasts (48). Together with our observation that miR$29 \mathrm{c}$ is downregulated by fibrotic ECM, these findings support a causal link between fibrotic ECM, downregulation of fibroblast miR-29, and the progression of fibrosis. Further study of how the cell-matrix interaction induces this miR-29 suppression could ultimately lead to potential targets for IPF treatment.

Supporting the importance of the cell-ECM interaction in IPF, the IPF ECM translationally activated the "integrin-binding" ontology (Table 1 and Supplemental Figure 2A). Moreover, when compiling all integrin-binding reactions from the Reactome data- 
A

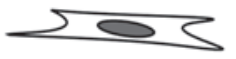

IPF fibroblasts scrambled control

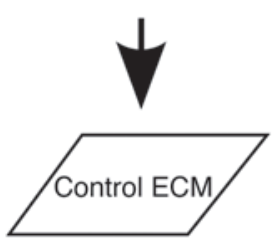

miR-29chi miR-29c targets ${ }^{10}$

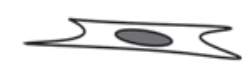

IPF fibroblasts scrambled control

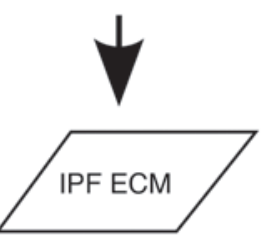

miR-29clo

miR-29c targets ${ }^{\text {hi }}$

C

CD276
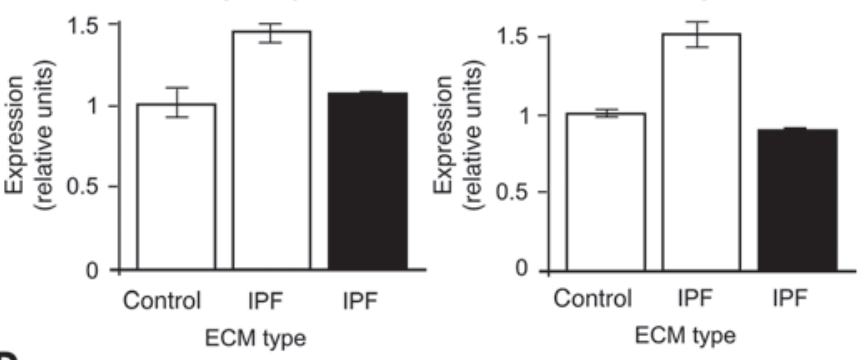

D
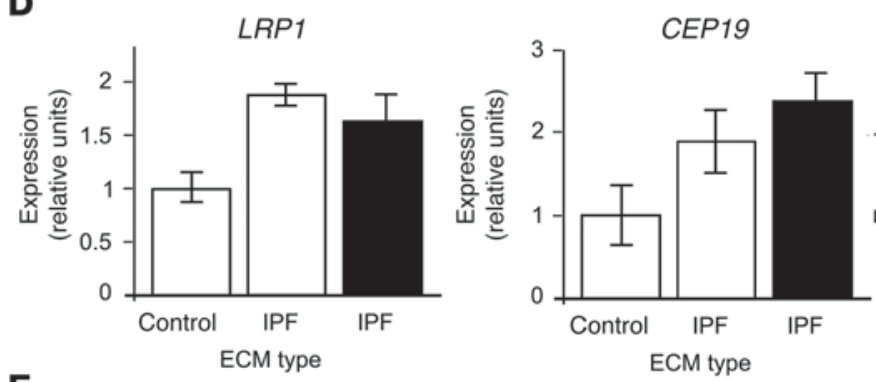

E

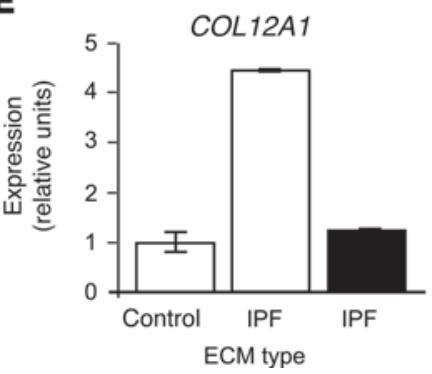

B

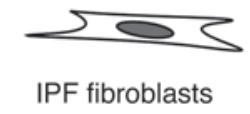
miR-29c ${ }^{+}$

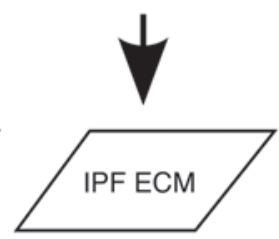

miR-29chi miR-29c targets ${ }^{10}$
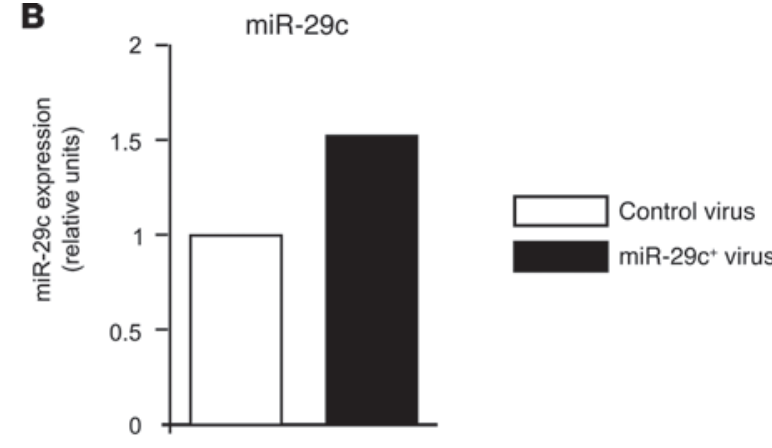

COL6A2

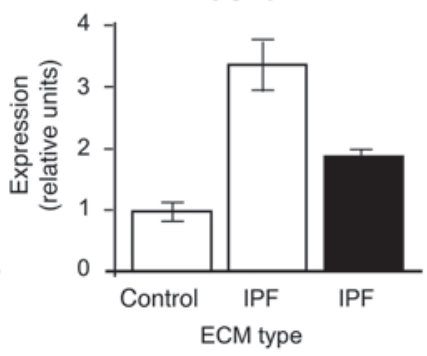

ZNF549

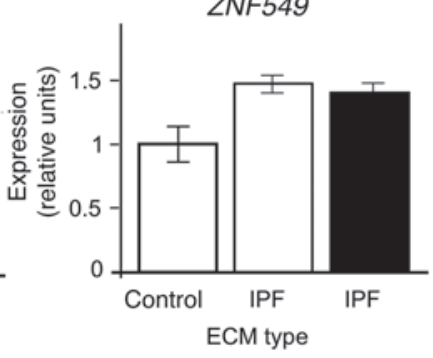

COL1A2

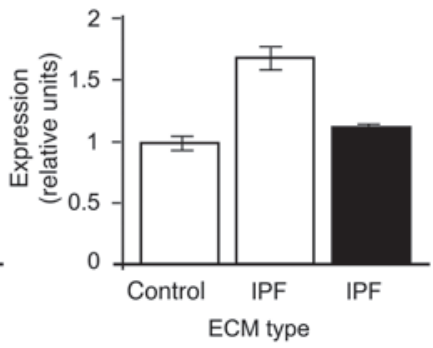

CHRNA5
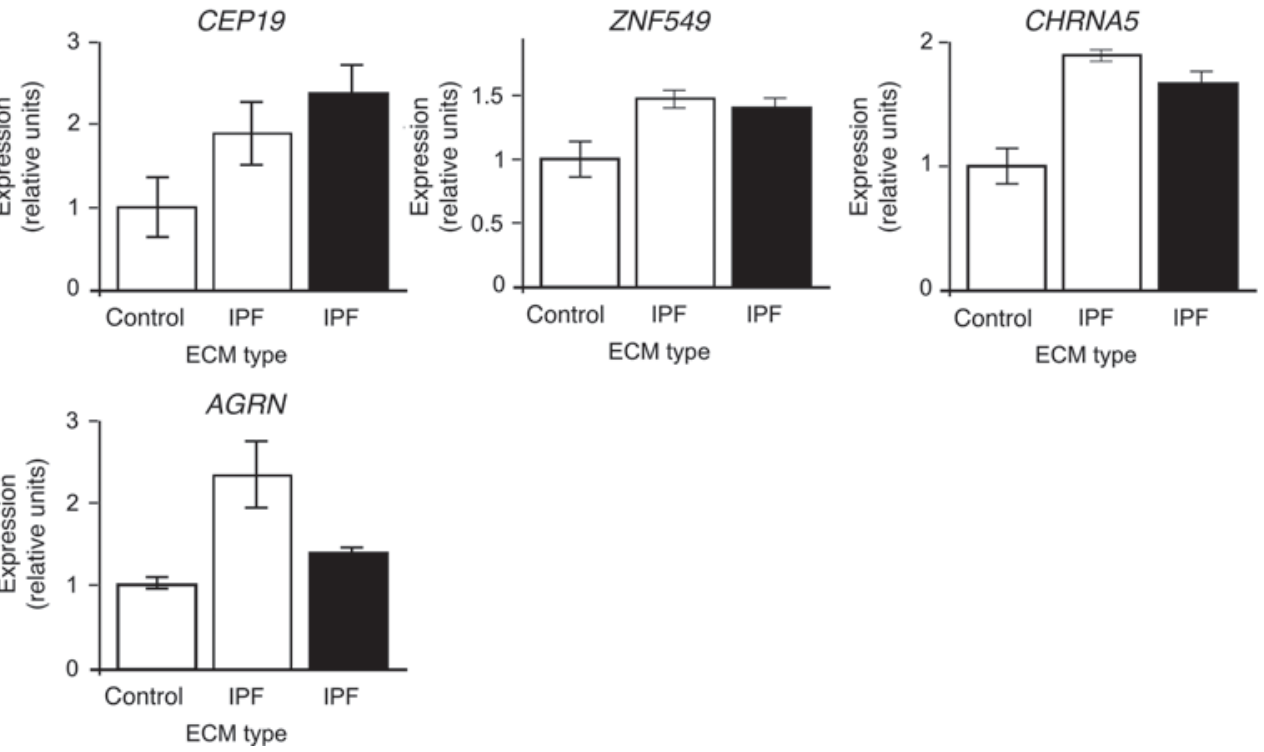

\section{Figure 6}

Overexpression of miR-29c abrogates pathological gene expression on IPF ECM. (A) Experimental design of miR-29c function study. (B) Relative expression of miR-29c in IPF cells treated with miR-29c+ virus or with scrambled control virus. (C) Relative expression of four genes containing miR-29 targets quantified using qPCR from polysome-associated RNA. White bars represent samples treated with control virus. Black bars represent samples treated with miR-29+ virus. Data represent the mean \pm SEM (three technical replicates). (D) Relative expression of four control genes. Controls were identified as genes that microarray indicated were upregulated by IPF but that did not contain miR-29 targets. (E) Relative expression of four ECM genes. ECM genes were identified as being upregulated by IPF but did not contain miR-29 targets.

base (49), in which both integrin subunits and at least one cognate ECM ligand were detected, the majority of reactions showed translational activation of the $\alpha$ integrin, the $\beta$ integrin, and the ligand(s), the exception being $\alpha_{4}$, which was unregulated (Supplemental Figure 2B). This further emphasizes that coordinated regulation of translation acts on ECM gene expression in response to IPF ECM.
This pattern of ECM-associated gene expression in our data raises an interesting question. Why are fibroblasts that are seeded into a collagen-rich ECM upregulating collagen, other ECM macromolecules, and their cognate integrin receptors? No previous study has shown IPF ECM to induce such an effect, nor has any study shown the antifibrotic miR-29 family to be downregulated by fibrotic ECM. This modulation provides evidence of a positive feedback loop 


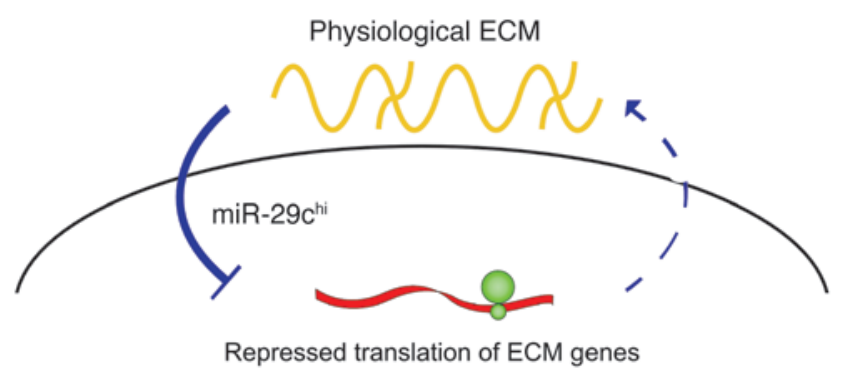

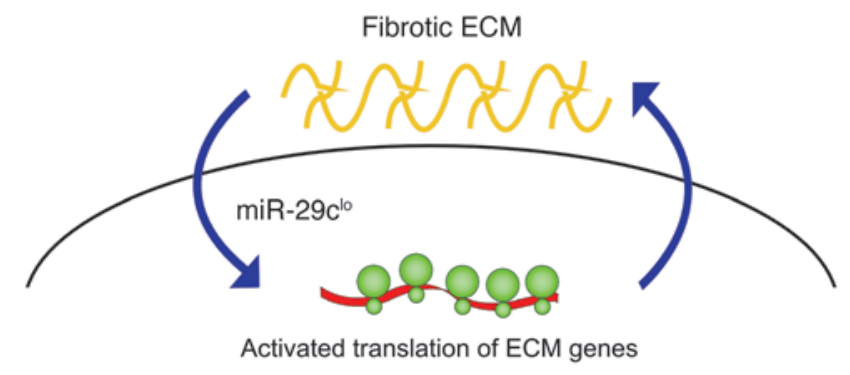

\section{Figure 7}

Positive feedback between the fibrotic ECM and the fibroblast amplifies the fibrotic phenotype. The IPF ECM induces translation of the genes that comprise the IPF ECM. This induces a positive feedback loop that amplifies ECM gene expression and spreads the fibrosis.

involving miR-29c whereby IPF ECM induces otherwise normal fibroblasts to produce additional ECM components (Figure 7). If this interpretation is correct, it suggests a model of IPF disease progression in which some initial insult creates a small fibrotic region in the lung. This fibrotic ECM corrupts nearby fibroblasts, which further remodel the surrounding lung tissue, spreading the fibrosis. Such a model would explain the contiguous nature of the fibrotic reticulum. It should be noted, however, that our data in no way preclude the possibility that subtle intrinsic changes to the fibroblast itself could also be important to disease initiation and progression. Indeed, we found that cell origin and ECM origin cooperatively regulated translation of a common set of ECM genes. We speculate that genetic or epigenetic changes to the fibroblast after what might be a physiological signal to heal is responsible for the initial fibrotic event and also prevents the cell from interrupting this feedback loop during disease progression. It remains to be determined whether targeting this feedback loop will be a way forward for the treatment of IPF.

\section{Methods}

Primary buman lung fibroblast cell lines. Human primary fibroblasts from ten different donors were used. These consisted of five control samples (mean age, 69 years) (histologically uninvolved lung distant from the resected tumor) and five samples from patients with IPF (mean age 63 years) (histologically confirmed usual interstitial pneumonitis). Tissue was obtained at the time of biopsy, autopsy, lung resection, or lung transplantation following procedures approved by the University of Minnesota Institutional Review Board for Human Subjects Research. The regions of lung chosen for IPF cells and ECM were documented by the pathologist of record to meet all criteria for usual interstitial pneumonitis, including active fibroblastic foci and did not represent areas that could be described as end-stage fibrosis. Lung tissue explants were cultivated in $35-\mathrm{mm}$ tissue culture dishes in explant medium (DMEM, 20\% FBS, $100 \mathrm{IU} / \mathrm{ml}$ penicillin, $100 \mathrm{IU} / \mathrm{ml}$ streptomycin, and $25 \mu \mathrm{g} / \mathrm{ml}$ amphotericin B) at $37^{\circ} \mathrm{C}$ in $95 \%$ air and $5 \%$ $\mathrm{CO}_{2}$. Outgrowth was evident in 5 to 7 days, and cells filled the dish in 2 to 3 weeks. Cells from each $35-\mathrm{mm}$ dish were released with trypsin-EDTA and expanded in 150-mm tissue culture dishes after trypsin was neutralized with fresh explant medium. These cells, designated passage 1, were cultivated in growth medium (DMEM,10\% FBS, $100 \mathrm{IU} / \mathrm{ml}$ penicillin, $100 \mathrm{IU} /$ $\mathrm{ml}$ streptomycin, and $25 \mu \mathrm{g} / \mathrm{ml}$ amphotericin B) at $37^{\circ} \mathrm{C}$ in $95 \%$ air and $5 \% \mathrm{CO}_{2}$. Medium was replaced twice weekly, and cells were subcultivated weekly at a 1:4 split ratio. Cells designated fibroblasts in both IPF and control samples had typical spindle morphology, were vimentin positive and $\alpha$ smooth muscle actin positive, and factor VIII negative and surfactant $\mathrm{C}$ negative. Cells between subcultivation numbers 4 and 7 were used in this study.
Decellularized primary human lung ECM. Pieces of lung tissue measuring approximately $30 \mathrm{~cm}^{3}$ were frozen at $-70^{\circ} \mathrm{C}$ and attached to 24 multiwell clusters filled with ice. The plate-adhered frozen tissue was allowed to equilibrate to a temperature of $-15^{\circ} \mathrm{C}$. A block plane was used to carefully make $200-\mu \mathrm{m}$ slices of frozen tissue, which were immediately placed in tissue culture dishes containing PBS and frozen in bulk at $-20^{\circ} \mathrm{C}$ for future use. When thawed, tissue slices were decellularized over the course of 4 days as follows: tissue slices were placed in a $100-\mathrm{mm}$ Petri dish, and $15 \mathrm{ml}$ of $1 \% \mathrm{SDS}$ in $\mathrm{H}_{2} \mathrm{O}$ (lysis solution) was added. Petri dishes were shaken at approximately 30 oscillations per minute on an orbital shaker at room temperature for 1 hour. Lysis solution was aspirated and replaced with $15 \mathrm{ml}$ of fresh lysis solution and placed again on the orbital shaker at the same settings. After an additional hour, lysis solution was replaced, and the tissue was shaken overnight at room temperature. Lysis solution was removed and replaced with $1 \%$ Triton $\mathrm{X}-100$ in $\mathrm{H}_{2} \mathrm{O}$ followed by three 1-hour cycles of agitation on an orbital shaker in replacement Triton X-100 solution. After treating with Triton X-100 overnight on an orbital shaker, tissue slices were rinsed twice with PBS and rinsed 8 times with deionized $\mathrm{H}_{2} \mathrm{O}$. To lyse any residual nuclei, $15 \mathrm{ml}$ of $1 \mathrm{M} \mathrm{NaCl}$ was added, and the tissue was placed on an orbital shaker at room temperature for 1 hour before being rinsed once with PBS and twice with deionized water. The tissue slices were treated with DNAase $(20 \mu \mathrm{g} / \mathrm{ml})$ plus $4.2 \mathrm{mM} \mathrm{MgCl}$ at $37^{\circ} \mathrm{C}$ for 1 hour. After aspirating the DNAase, the tissue was rinsed twice with deionized water and once with PBS plus antibiotics for 30 minutes at room temperature on an orbital shaker. The PBS was removed and replaced with $20 \mathrm{ml}$ of complete medium (DMEM, 10\% FBS, and $300 \mathrm{IU} / \mathrm{ml}$ penicillin, $300 \mathrm{IU} / \mathrm{ml}$ streptomycin, and $75 \mu \mathrm{g} / \mathrm{ml}$ amphotericin B) and shaken overnight at room temperature. This decellularization procedure is similar to a previously described procedure (50). Tissue slices were incubated $\left(37^{\circ} \mathrm{C}, 5 \% \mathrm{CO}_{2}\right)$ for 1 hour prior to seeding with cells. Cultured fibroblasts were released from tissue culture dishes with $0.25 \%$ trypsin/EDTA, centrifuged for 5 minutes at $900 \mathrm{~g}$, and resuspended in complete medium at a concentration of $10^{6}$ cells $/ \mathrm{ml}$. Each tissue slice was placed in a $50-\mathrm{ml}$ conical tube with IPF or control fibroblasts $\left(10^{6} \mathrm{cells} / \mathrm{cm}^{2}\right.$ of decellularized $\mathrm{ECM})$. The conical tubes containing tissue slices and cells were gently rotated at 1 oscillation per minute in a humidified $37^{\circ} \mathrm{C} / 10 \% \mathrm{CO}_{2}$ incubator for 18 hours with no media changes. In order to minimize the effect of variance induced by running samples at different times (batch variance), each control cell line was processed in tandem with an IPF cell line.

Following incubation, cycloheximide was added to the media at a final concentration of $100 \mu \mathrm{g} / \mathrm{ml}$ to immobilize ribosomes on RNA. Tissues were returned to the incubator for 5 minutes. The tissue slices were rinsed thoroughly in PBS containing cycloheximide to remove nonadherent cells. The slices were blotted semi-dry, flash-frozen in liquid nitrogen, and stored at $-70^{\circ} \mathrm{C}$. 
RNA isolation and polysome preparations. A mortar and pestle set was placed into liquid nitrogen to equilibrate the temperature. Flash-frozen tissue was removed from the freezer and placed into the mortar. Tissue was ground to a fine powder and scraped into a liquid nitrogen-cooled microcentrifuge tube. A portion of the powder approximating $5 \%$ of the total material was placed into a microcentrifuge tube containing $500 \mu \mathrm{l}$ of Tri-reagent (Sigma-Aldrich) and $100 \mu \mathrm{l}$ of chloroform. RNA was isolated and precipitated using isopropanol according to the manufacturer's instructions.

The remainder of the frozen powder was added to an ice-cooled 7-ml Dounce homogenizer. Swelling buffer $(375 \mu \mathrm{l})$ was immediately added $(10 \mathrm{mM} \mathrm{NaCl}, 20 \mathrm{mM}$ Tris, $\mathrm{pH} 7.5,3 \mathrm{mM} \mathrm{MgCl}$ ) and supplemented with $200 \mathrm{U}$ of Superase-In RNase Inhibitor (Thermo Fisher Scientific) and DTT (1 mM final concentration). Lysis buffer (125 ml; $0.2 \mathrm{M}$ sucrose and $1.2 \%$ Triton X-100 in low-salt buffer [LSB]) was added, and the homogenate was churned 15 times with a tight-fitting pestle. The homogenate was transferred to a microcentrifuge tube and centrifuged at $16,000 \mathrm{~g}$ for $1 \mathrm{minute}$. The clarified supernatant was transferred to a clean tube containing $100 \mathrm{U}$ of Superase-In and centrifuged at $16,000 \mathrm{~g}$ for an additional 3 minutes. The supernatant was transferred to a tube containing $15 \mu \mathrm{l}$ of $5 \mathrm{M} \mathrm{NaCl}$ and $60 \mathrm{U}$ of Superase-In RNase and layered onto a 5-ml, $0.5-1.5 \mathrm{M}$ sucrose gradient, which was centrifuged at $200,000 \mathrm{~g}$ in a Beckman Coulter SW55Ti rotor for 80 minutes at $4^{\circ} \mathrm{C}$. The RNA was fractionated and prepared as previously described (51). Ten fractions of $0.5 \mathrm{ml}$ were collected into baked glass tubes containing $50 \mu \mathrm{l}$ of $10 \%$ SDS. RNA in each fraction was purified using Tri-reagent, and fractions $7-10$ were combined into the actively translated heavy sample ( $>3$ ribosomes per transcript).

Proteomic measurement of IPF lung tissue has been previously described (38).

Microarray analysis. Total and heavy RNA samples were submitted to the University of Minnesota Biomedical Genomics Center for cDNA labeling and microarray analysis using the Illumina Expression BeadChip system. Bead summary data were normalized using the robust spline normalization and $\log _{2}$ transformed in R/Bioconductor. Probes for which the expression level exceeded all negative controls (detection $P$ value $=0.0$ ) in 2 of 3 of steady-state samples were included in downstream analysis. Gene expression levels were mapped from probe levels by selecting the highest expressing probe from each mapped gene.

The experiment is a split-plot design, with cell origin and processing batch as between-subject factors and ECM type as a within-subject factor. Therefore, to analyze the cell-based effects, the expression values for both ECM types (control and IPF) were averaged across both ECM types for each cell line for each gene. To probe for expression differences in the levels of polysome and total steady-state RNA, the following per-gene linear model was fit:

$\left(E x_{I P F}+E x_{\text {control }}\right) / 2=\gamma_{\text {batch }}+\gamma_{\text {cell-origin }}+\varepsilon_{\text {between-subjects }}$

\section{(Equation 2)}

where $E x_{I P F}$ and $E x_{\text {control }}$ are the expression of a particular cell line on IPF and control ECM, respectively (averaged to probe the cell origin differences), $\gamma_{\text {batch }}$ accounts for variance among sample batches, $\gamma_{\text {cell-origin }}$ is the expression difference between IPF and control fibroblasts, and $\varepsilon_{\text {between-subjects }}$ is the between-subjects error.

ECM effects were then analyzed in a paired design: each cell line was cultured on both ECM types. Note, since for each cell line both ECM types were processed simultaneously, the processing batch is not included in the model.
$E x=\gamma_{E C M}+\gamma_{\text {cell line }}+\varepsilon_{\text {within-subject }}$

(Equation 3)

where $E x$ is the expression, $\gamma_{E C M}$ is the effect of IPF ECM, $\gamma_{\text {cell line }}$ is the expression of the gene in a particular cell line (included to pair the design), and $\varepsilon_{\text {within-subject }}$ is the within-subject error.

To probe specifically for differences in the level of ribosome recruitment (translation), the polysome-associated RNA levels must be decoupled from the measurement of total steady-state RNA. In order to do this, the models were extended to include a linear fit to the total steady-state RNA levels. For cell-type effects, this yielded:

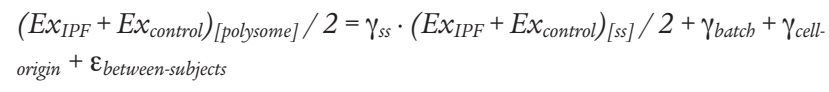

(Equation 4)

where $\gamma_{s s}$ is the fitted linear dependence of the total steady-state RNA expression level on the polysome-associated expression level. Similarly, for analysis of the ECM effects, the model fitted was:

$E x_{[p o l y s o m e]}=\gamma_{s s} \cdot E x_{[s s]}+\gamma_{E C M}+\gamma_{\text {cell line }}+\varepsilon_{\text {within-subject }}$

\section{(Equation 5)}

where again a paired design was used. All statistics were calculated using the "car" R package (52). All $P$ values were adjusted using random variance model (RVM) statistics (53). Gene set enrichment analysis was performed using the GAGE R package (37) on a preranked list of genes (according to their signed $-\log _{10} P$ values) and ontologies from the Gene Ontology Consortium (54).

Genes targeted by miR-29 were those predicted by TargetScanHuman by the presence of a 7 -mer or 8-mer site matching the target region of the miR-29abcd family (55). The microarray data from this publication have been submitted to the Gene Expression Omnibus (GEO) database (GEO GSE45686).

External datasets. External datasets (GEO GSE17978, GSE10921, and GSE10667) were acquired through the GEO. Statistics were calculated from the obtained data using an equal variance Student's $t$ test. The obtained $P$ values were corrected using RVM.

To check for coherence among external datasets $(29,30)$ and our current data, in each comparison genes were divided into four categories: those genes upregulated in IPF in both the external dataset and our data, genes downregulated in both datasets, genes upregulated in the external data and downregulated here, and genes downregulated here but upregulated in the external dataset. The resulting contingency tables were tested for agreement using Cohen's $\kappa$ coefficient.

miR quantification. qPCR was performed on total RNA samples for each of the cell/ECM conditions using the miScript SYBR Green PCR kit (QIAGEN) according to the manufacturer's instructions. qPCR was performed for miR-29a, miR-29b, and miR-29c using prevalidated primers from the kits. Amplification was allowed to proceed for 40 cycles at which time product was quantified at the log-linear portion of the curve using LightCycler analysis software.

Gain-of-miR-29c-function. A lentivirus plasmid expressing hsa-miR29c (PMIRH29c-PA1) was obtained from System Biosciences. HEK293T/c17 cells were purchased from ATCC and cultured in DMEM with 10\% FBS and antibiotics (penicillin $100 \mathrm{IU} / \mathrm{ml}$, streptomycin $100 \mathrm{IU} / \mathrm{ml}$, and amphotericin B $25 \mu \mathrm{g} / \mathrm{ml}$ ). Third-generation lentiviral packaging plasmids 
pMDLg/RRE and pRSV-REV were obtained from Addgene. For the generation of VSV-G pseudotyped lentivirus pMIRH29c $(9.3 \mu \mathrm{g})$ along with pMD2.G $(2.9 \mu \mathrm{g}), \mathrm{pMDLg} / \mathrm{RRE}(5.4 \mu \mathrm{g})$ and pRSV-REV $(2.3 \mu \mathrm{g})$ were cotransfected into $10-\mathrm{cm}$ dishes of subconfluent HEK293T/c17 cells using Fugene $6(60 \mu \mathrm{l})(56-61)$. DNA complexes were incubated with the cells for 4 hours $\left(37^{\circ} \mathrm{C}, 5 \% \mathrm{CO}_{2}\right)$. Medium was removed and replaced with $5 \mathrm{ml}$ DMEM, $10 \% \mathrm{FBS}$, and antibiotic medium containing $2 \mathrm{mM}$ caffeine (62). The transfected cells were incubated for 24 hours $\left(37^{\circ} \mathrm{C}, 5 \% \mathrm{CO}_{2}\right)$, and media were removed and replaced with $5 \mathrm{ml} \mathrm{DMEM}, 10 \% \mathrm{FBS}$, and antibiotic medium containing $2 \mathrm{mM}$ caffeine. Viral supernatants were harvested 48 hours after transfection, filtered through a $0.45-\mu \mathrm{m}$-pore-size PVDF filter, aliquoted, and frozen at $-80^{\circ} \mathrm{C}$. Target cells were plated in 6-well dishes and allowed to attach overnight. Virus was added to cells in DMEM plus $10 \%$ FBS with polybrene at a final concentration of $8 \mu \mathrm{g} / \mathrm{ml}$, and the plate was centrifuged $\left(1,200 \mathrm{~g}, 25^{\circ} \mathrm{C}\right)$ for 1 hour and incubated for 16 hours $\left(37^{\circ} \mathrm{C}, 5 \% \mathrm{CO}_{2}\right)$. Virus was removed, $2 \mathrm{ml}$ fresh medium per well was added, and incubation was continued until the cells were used for experiments.

qPCR. Low-passage IPF cells from a single patient were grown in 6-well cluster plates to $80 \%$ confluence. Medium was aspirated and replaced with either miR29c lentivirus medium or control lentivirus medium diluted 1:4 in growth medium supplemented with polybrene $(8 \mu \mathrm{g} / \mathrm{ml})$. The dishes were centrifuged for 60 minutes at $1,000 \mathrm{~g}$ and incubated for 24 hours, and medium was replaced with growth medium. Cells were expanded for two passages, and total RNA was collected to perform qPCR to assess miR29c levels as described in Methods. Cells were expanded for an additional passage, and the IPF cells harboring control virus were collected and cultured on IPF and control matrices, while the miR-29c virally transduced cells were cultured on IPF matrix as described in Methods. Cultures were maintained for 18 hours, and the cell/matrix samples were collected and processed for polyribosome preparations as described in Methods. RNA was collected from the translationally active fractions 7-10 and reverse transcribed using a TaqMan Reverse Transcription kit (Roche). qPCR was performed on the products for four of the matrix-associated genes iden- tified in our microarray experiments. The following primer sets were generated by the University of Minnesota Genomics Center: CD276 forward: 5'-GCAGTCTTTTCCTGGCTTGC-3' , reverse: 5'-GCAGGGAGAGATGACATCCG-3'; TMEM132A forward: 5'-TGCGTGGCCATCTTCATCTT-3', reverse: 5'-ACTGTCGGGAGGTTCTTTGC-3'; COL6A2 forward: 5'-AGCCTACGGAGAGTGCTACA-3', reverse: 5'-GTCCTGGGAATCCAATGGGG-3'; COL1A2 forward: 5'-CAAGGTTTCCAAGGACCTGC-3', reverse: 5'-CССТTCAATCCATCCAGACCA-3'; GAPDH forward: 5'-TGCACCACCAACTGCTTAGC-3', reverse: 5'-GGCATGGACTGTGGTCATGAG-3'.

qPCR was performed for 40 cycles using a LightCycler FastStart DNA MasterPLUS SYBR Green I Kit (Roche) in a LightCycler 1.5 (Roche). Samples were quantified at the log-linear portion of the curve using LightCycler analysis software and normalized to GAPDH.

Study approval. This study involved the development of cell lines from human lung tissue provided by the University of Minnesota Tissue Procurement Service. The procedure was approved by the IRB of the University of Minnesota.

\section{Acknowledgments}

This work was supported by NIH grants P01 HL91775 (to C.A. Henke), R01 HL089249 (to P.B. Bitterman), and F32 HL116105 (to M.W. Parker); by funds provided by the O'Brien family and the Carlson Family Fund (to M.W. Parker); and by the Swedish Research Council and the Swedish Cancer Foundation (to O. Larsson). E.S. White was supported by NIH grant U01 HL111016.

Received for publication June 3, 2013, and accepted in revised form December 27, 2013.

Address correspondence to: Matthew W. Parker, 420 Delaware St. SE, Pulmonary MMC: 276, Minneapolis, Minnesota 55455, USA. Phone: 612.626.1124; Fax: 612.625.2174; E-mail: park0523@umn.edu.
1. Halliday NL, Tomasek JJ. Mechanical properties of the extracellular matrix influence fibronectin fibril assembly in vitro. Exp Cell Res. 1995;217(1):109-117.

2. Pelham RJ Jr, Wang Y. Cell locomotion and focal adhesions are regulated by the mechanical properties of the substrate. Biol Bull. 1998;194(3):348-350.

3. Rhudy RW, McPherson JM. Influence of the extracellular matrix on the proliferative response of human skin fibroblasts to serum and purified platelet-derived growth factor. J Cell Physiol. 1988;137(1):185-191.

4. Schor SL. Cell proliferation and migration on collagen substrata in vitro. J Cell Sci. 1980; 41(1):159-175.

5. Bhowmick NA, Neilson EG, Moses HL. Stromal fibroblasts in cancer initiation and progression. Nature 2004;432(7015):332-337.

6. Kalluri R, Zeisberg M. Fibroblasts in cancer. Nat Rev. 2006;6(5):392-401.

7. Wiseman BS, Werb Z. Stromal effects on mammary gland development and breast cancer. Science 2002;296(5570):1046-1049.

8. Wynn TA, Ramalingam TR. Mechanisms of fibrosis: therapeutic translation for fibrotic disease. Nat Med. 2012;18(7):1028-1040.

9. Hinz B, et al. Recent developments in myofibroblast biology: paradigms for connective tissue remodeling. Am J Pathol. 2012;180(4):1340-1355.

10. Selman $\mathrm{M}$, et al. Idiopathic pulmonary fibrosis: prevailing and evolving hypotheses about its pathogenesis and implications for therapy. Ann Intern Med. 2001;134(2):136-151.

11. Noble PW, et al. Idiopathic pulmonary fibrosis: new insights into pathogenesis. Clin Chest Med.
2004;25(4):749-758.

12. King TE, Pardo A, Selman M. Idiopathic pulmonary fibrosis. Lancet. 2011;378(9807):1949-1961.

13. Cool CD, Groshong SD, Rai PR, Henson PM, Stewart JS, Brown KK. Fibroblast foci are not discrete sites of lung injury or repair the fibroblast reticulum. Am J Respir Crit Care Med. 2006;174(6):654-658.

14. Agustí AG, Roca J, Gea J, Wagner PD, Xaubet A, Rodriguez-Roisin R. Mechanisms of gas-exchange impairment in idiopathic pulmonary fibrosis. Am J Respir Crit Care Med. 1991;143(2):219-225.

15. Noble PW. Idiopathic pulmonary fibrosis: natural history and prognosis. Clin Chest Med. 2006;27(1):S11-S16.

16. Frankel SK, et al. Tnf- $\alpha$ sensitizes normal and fibrotic human lung fibroblasts to fas-induced apoptosis. Am J Respir Cell Mol Biol. 2006; 34(3):293-304.

17. Moodley YP, et al. Fibroblasts isolated from normal lungs and those with idiopathic pulmonary fibrosis differ in interleukin-6/gp130-mediated cell signaling and proliferation. Am J Pathol. 2003;163(1):345-354.

18. Ramos C, et al. Fibroblasts from idiopathic pulmonary fibrosis and normal lungs differ in growth rate, apoptosis, and tissue inhibitor of metalloproteinases expression. Am J Respir Cell Mol Biol. 2001;24(5):591-598.

19. Xia $\mathrm{H}$, et al. Pathological integrin signaling enhances proliferation of primary lung fibroblasts from patients with idiopathic pulmonary fibrosis. J Exp Med. 2008;205(7):1659-1672.

20. Allen JT, Spiteri MA. Growth factors in idiopathic pulmonary fibrosis: relative roles. Respir Res.
2001;3:13.

21. McKeown S, Richter AG, O'Kane C, McAuley DF, Thickett D. Mmp expression and abnormal lung permeability are important determinants of outcome in ipf. Eur Respir J. 2009;33(1):77-84.

22. Pilewski JM, Liu L, Henry AC, Knauer AV, FeghaliBostwick CA. Insulin-like growth factor binding proteins 3 and 5 are overexpressed in idiopathic pulmonary fibrosis and contribute to extracellular matrix deposition. Am J Pathol. 2005;166(2):399-407.

23. Thannickal VJ, Horowitz JC. Evolving concepts of apoptosis in idiopathic pulmonary fibrosis. Proc Am Thorac Soc. 2006;3(4):350-356.

24. Frantz C, Stewart KM, Weaver VM. The extracellular matrix at a glance. J Cell Sci. 2010;123(24):4195-4200.

25. MacKenna D, Summerour SR, Villarreal FJ. Role of mechanical factors in modulating cardiac fibroblast function and extracellular matrix synthesis. Cardiovasc Res. 2000;46(2):257-263.

26. Zhou Y, et al. Inhibition of mechanosensitive signaling in myofibroblasts ameliorates experimental pulmonary fibrosis. J Clin Invest. 2013;123(3):1096-1108.

27. Larsson O, Tian B, Sonenberg N. Toward a genome-wide landscape of translational control. Cold Spring Harb Perspect Biol. 2013;5(1):a012302.

28. Larsson O, et al. Fibrotic myofibroblasts manifest genome-wide derangements of translational control. PLoS One 2008;3(9): e3220.

29. Emblom-Callahan M, et al. Genomic phenotype of non-cultured pulmonary fibroblasts in idiopathic pulmonary fibrosis. Genomics 2010;96(3):134-145.

30. Konishi K, et al. Gene expression profiles of acute exacerbations of idiopathic pulmonary fibrosis. Am J Respir Crit Care Med. 2009;180(2):167-175. 
31. Ingolia NT, Ghaemmaghami S, Newman JR, Weissman JS. Genome-wide analysis in vivo of translation with nucleotide resolution using ribosome profiling. Science 2009;324(5924):218-223.

32. Benjamini Y, Hochberg Y. Controlling the false discovery rate: a practical and powerful approach to multiple testing. J R Stat Soc Ser B Stat Methodol. 1995;57(1):289-300.

33. Vuga L, et al. Wnt5a is a regulator of fibroblast proliferation and resistance to apoptosis. Am J Respir Cell Mol Biol. 2009;41(5):583-589.

34. Larsson O, Sonenberg N, Nadon R. Identification of differential translation in genome wide studies. Proc Natl Acad Sci U S A. 2010;107(50):21487-21492.

35. Larsson O, Sonenberg N, Nadon R. anota: analysis of differential translation in genome-wide studies. Bioinformatics 2011;27(10):1440-1441.

36. Colman H, et al. Genome-wide analysis of host mrna translation during hepatitis $\mathrm{c}$ virus infection. J Virol. 2013;87(12):6668-6677.

37. Luo W, Friedman M, Shedden K, Hankenson K, Woolf P. Gage: generally applicable gene set enrichment for pathway analysis. BMC Bioinformatics. 2009; 10:161.

38. Booth A, et al. Acellular normal and fibrotic human lung matrices as a culture system for in vitro investigation. Am J Respir Crit Care Med. 2012;186(9):866-876.

39. Sonenberg N, Hinnebusch AG. Regulation of translation initiation in eukaryotes: mechanisms and biological targets. Cell. 2009;136(4):731-745.

40. Cushing L, et al. mir-29 is a major regulator of genes associated with pulmonary fibrosis. Am J Respir Cell Mol Biol. 2011;45(2):287-294.

41. Pandit K, Milosevic J, Kaminski N. Micrornas in idiopathic pulmonary fibrosis. Transl Res. 2011;157(4):191-199.
42. Schwanhäusser B, et al. Global quantification of mammalian gene expression control. Nature. 2011;473(7347):337-342.

43. Oak SR, et al. A micro rna processing defect in rapidly progressing idiopathic pulmonary fibrosis. PloS One. 2011;6(6):e21253.

44. Maurer B, et al. Microrna-29, a key regulator of collagen expression in systemic sclerosis. Arthritis Rheum. 2010;62(6):1733-1743.

45. Qin W, et al. Tgf- $\beta / \operatorname{smad} 3$ signaling promotes renal fibrosis by inhibiting mir-29. J Am Soc Nephrol. 2011;22(8):1462-1474

46. Van Rooij E, et al. Dysregulation of micrornas after myocardial infarction reveals a role of mir29 in cardiac fibrosis. Proc Natl Acad Sci U S A. 2008;105(35):13027-13032.

47. Xiao J, et al. mir-29 inhibits bleomycin-induced pulmonary fibrosis in mice. Mol Ther. 2012; 20(6):1251-1260.

48. Yang $T$, et al. Mir- 29 mediates tgf $\beta 1$-induced extracellular matrix synthesis through activation of pi3k-akt pathway in human lung fibroblasts. J Cell Biochem. 2013;114(6):1336-1342.

49. Matthews L, et al. Reactome knowledgebase of human biological pathways and processes. Nucleic Acids Res. 2009;37(suppl 1):D619-D622.

50. Lwebuga-Mukasa JS, Ingbar DH, Madri JA. Repopulation of a human alveolar matrix by adult rat type ii pneumocytes in vitro: a novel system for type ii pneumocyte culture. Exp Cell Res. 1986;162(2):423-435.

51. Li S, et al. Translation factor eif4e rescues cells from myc-dependent apoptosis by inhibiting cytochromec release. J Biol Chem. 2003;278(5):30153022 .

52. Weisberg HS, Fox J. An R Companion To Applied Regression. 2nd ed. Thousand Oaks, California,
USA: Sage Publications, Inc.; 2010.

53. Wright GW, Simon RM. A random variance model for detection of differential gene expression in small microarray experiments. Bioinformatics 2003;19(18):2448-2455.

54. Ashburner M, et al. Gene ontology: tool for the unification of biology. Nat Genet. 2000;25(1):25-29.

55. Lewis BP, Burge CB, Bartel DP. Conserved seed pairing, often flanked by adenosines, indicates that thousands of human genes are microrna targets. Cell. 2005;120(1):15-20.

56. Chen C, Okayama H. Calcium phosphate-mediated gene transfer: a highly efficient transfection system for stably transforming cells with plasmid dna. Biotechniques. 1987;6(7):632-638.

57. Miyoshi H, Blömer U, Takahashi M, Gage FH, Verma IM. Development of a self-inactivating lentivirus vector. J Virol. 1998;72(10):8150-8157.

58. Mochizuki H, Schwartz JP, Tanaka K, Brady RO, Reiser J. High-titer human immunodeficiency virus type 1-based vector systems for gene delivery into nondividing cells. J Virol. 1998;72(11):8873-8883.

59. Burns JC, Friedmann T, Driever W, Burrascano M, Yee JK. Vesicular stomatitis virus g glycoprotein pseudotyped retroviral vectors: concentration to very high titer and efficient gene transfer into mammalian and nonmammalian cells. Proc Natl Acad SciUS A. 1993;90(17):8033-8037.

60. Zufferey R, et al. Self-inactivating lentivirus vector for safe and efficient in vivo gene delivery. J Virol. 1998;72(12):9873-9880.

61. Dull T, et al. A third-generation lentivirus vector with a conditional packaging system. J Virol. 1998;72(11):8463-8471.

62. Ellis BL, Potts PR, Porteus MH. Creating higher titer lentivirus with caffeine. Hum Gene Ther. 2010;22(1):93-100. 